\title{
STEADY-STATE ENTANGLEMENT BY ENGINEERED QUASI-LOCAL MARKOVIAN DISSIPATION
}

\author{
Francesco Ticozzi* \\ Dipartimento di Ingegneria dell'Informazione, Università di Padova, via Gradenigo 6/B, 35131 Padova, Italy and \\ Department of Physics and Astronomy, Dartmouth College, 6127 Wilder Laboratory, Hanover, NH 03755, USA \\ Lorenza Viold \\ Department of Physics and Astronomy, Dartmouth College, 6127 Wilder Laboratory, Hanover, NH 03755, USA
}

(Dated: November 2, 2018)

\begin{abstract}
We characterize and construct time-independent Markovian dynamics that drive a finitedimensional multipartite quantum system into a target (pure) entangled steady state, subject to physical locality constraints. In situations where the desired stabilization task can not be attained solely based on local dissipative means, we allow for local Hamiltonian control or, if the latter is not an option, we suitably restrict the set of admissible initial states. In both cases, we provide algorithms for constructing a master equation that achieves the intended objective and show how this can genuinely extend the manifold of stabilizable states. In particular, we present quasi-local control protocols for dissipatively engineering multipartite GHZ "cat" states and W states on $n$ qubits. For GHZ states, we find that no scalable procedure exists for achieving stabilization from arbitrary initial states, whereas this is possible for a target W state by a suitable combination of a two-body Hamiltonian and dissipators. Interestingly, for both entanglement classes, we show that quasi-local stabilization may be scalably achieved conditional to initialization of the system in a large, appropriately chosen subspace.
\end{abstract}

PACS numbers:

Keywords: Quantum control, engineered dissipation, entanglement, quantum dynamical semigroups.

\section{INTRODUCTION}

Generating and manipulating highly non-classical entangled states in a robust scalable fashion is a central goal across Quantum Information Processing (QIP) [1] and quantum engineering [2]. While traditional circuit-based schemes are based on the application of suitable coherent control, implementing a desired sequence of quantum gates on a known fiducial state, progress in controlling open quantum systems is prompting a systematic exploration of incoherent control schemes using engineered dissipation. Access to dissipative control parameters, alone or in conjunction with Hamiltonian ones, may generally allow for enhanced efficiency and added flexibility in achieving relevant QIP tasks. Notably, quantum simulation of arbitrary open-system dynamics on a quantum computer may be accomplished by suitable dissipative control on just one more "ancillary" qubit than needed to simulate closed-system dynamics 3], as demonstrated in recent trapped-ion experiments [4, 5] (see also [6]). Likewise, dissipative quantum-state preparation protocols may allow for arbitrary initial states to be driven into a desired target state by "all-to-one" control laws that have no counterpart for unitary evolutions, with the

\footnotetext{
*Electronic address: ticozzi@dei.unipd.it

${ }^{\dagger}$ Electronic address: lorenza.viola@dartmouth.edu
}

potential for inherent robustness against transient perturbations. As a result, schemes for dissipative quantum control and generation of entangled states of light and matter via "reservoir engineering" have been extensively investigated theoretically in different contexts, see e.g. [7-25].

As control methodologies that rely on engineered dissipation become increasingly attractive experimentally [4, 26 28], it is essential to develop rigorous controltheoretic characterizations of the set of target states achievable in open quantum systems given realistic control resources, and to devise constructive control design protocols. In this context, an important task is provided by dissipative entanglement engineering in multipartite systems evolving according to a continuous-time quantum Markov process [29]. It is worth stressing that designing Markovian dynamics that admits a desired (pure or mixed) target state as its unique steady state (that is, achieves global asymptotic stability at the target) is relatively straightforward in the absence of control constraints, and different constructive strategies exist. In fact, application of a single time-independent Hamiltonian and Markovian noise channel suffices in principle in the generic case [16]. Alternatively, if the system is completely controllable in the absence of dissipation and the control actions can be enacted fast with respect to the noise time-scales, it clearly suffices to identify a purely dissipative dynamics that stabilizes any state with the same spectrum as the target, followed by rapid unitary control in order to suitably rotate such state into the target [18].

In practice, however, physical evolutions are not de- 
scribed by arbitrary Markovian master equations, and available coherent and incoherent controls are inevitably constrained. In particular, physically admissible Hamiltonian and noise (Lindblad) operators are typically described by operators that act non-trivially on finite subsets of subsystems, that is, are Quasi-Local (QL) relative to the given tensor-product decomposition. Necessary and sufficient conditions for a pure entangled state to be stabilizable in the absence of pre-existing "drift" dynamics under QL constraints have been obtained in [36], assuming purely dissipative means (so-called Dissipative QL Stabilization, DQLS for short).

In this work, we continue and substantially expand our investigation, with the twofold goal of (i) obtaining a complete characterization of the DQLS setting in the presence of non-trivial drift dynamics [37]; (ii) defining and analyzing alternative QL settings, which may allow for a desired stabilization task to be achieved in cases where purely dissipative control is insufficient. While we shall in general allow for both time-independent dissipative and Hamiltonian (coherent) control resources, no access to a universal set of tunable Hamiltonians nor auxiliary quantum resources will be assumed, preventing switching schemes along the lines envisioned in [18] or 24] from being viable. Specifically, two natural complementary strategies will be pursued: to either augment the available control resources, by suitably combining (timeindependent) QL Hamiltonian control with dissipation; or, if Hamiltonian control is not an option or is otherwise unfeasible, to insist on purely dissipative stabilization by restricting the initial condition to an appropriately chosen "attraction basin".

The content is organized as follows. We begin in Section IA by introducing the relevant class of dynamical models and different QL stabilization settings, along with required known results on DQLS states. Hamiltonianassisted and conditional QL stabilization are first analyzed in Section [II] and Section [IV] respectively, under the simplifying assumption that internal drift dynamics is absent or can be ignored. In both cases, we develop necessary conditions that the control Hamiltonian or, respectively, the initialization subspace must obey in order for the target state to be QL stabilizable, and then present a constructive randomized algorithm that outputs a choice of stabilizing controls with unit probability. We reconsider and address the role of underlying Markovian drift dynamics in Section V identifying necessary conditions for the desired stabilization to be compatible with the drift, and showing how different scenarios may then be tackled by adapting our previous results. Section VI present explicit QL stabilization protocols for dissipative engineering of GHZ and $\mathrm{W}$ states in $n$-qubit systems. While none of these highly entangled states can be generated solely based on QL dissipative means, except for trivial exceptions [36], we show that for both classes QL stabilization is achievable in a scalable fashion for a large subspace of initial states. For GHZ states, we additionally establish that no scalable procedure exists for achiev- ing stabilization from arbitrary initial states, whereas arbitrary initial states may be driven to a target $\mathrm{W}$ state by a suitable combination of two-body Hamiltonian and dissipators. Concluding remarks are given in Section VII. We include in separate Appendices the proof of convergence of our randomized construction as well as, for completeness and direct reference, an algorithm for verifying global stability, originally developed in [35], that is required in the proof.

\section{PRELIMINARIES AND BACKGROUND}

\section{A. Quasi-local quantum dynamical semigroups}

We consider a finite-dimensional multipartite open quantum system $S$ consisting of $n$ (distinguishable) subsystems, defined on a tensor-product Hilbert space

$\mathcal{H}=\bigotimes_{a=1}^{n} \mathcal{H}_{a}, \quad a=1, \ldots, n, \quad \operatorname{dim}\left(\mathcal{H}_{a}\right)=d_{a}, \operatorname{dim}(\mathcal{H})=d$

Let $\mathfrak{D}(\mathcal{H})$ denote the (convex) set of physical states (density operators) of $S$, that is, trace-one, positive semidefinite operators with support on $\mathcal{H}$. In analogy with probability distributions, we shall denote the support of $\rho \in \mathfrak{D}(\mathcal{H})$ by $\operatorname{supp}(\rho)=\operatorname{range}(\rho)$. It is then natural to use $\mathfrak{D}\left(\mathcal{H}^{\prime}\right)$ to describe the set of density operators with support contained in a subspace $\mathcal{H}^{\prime} \subseteq \mathcal{H}$.

In many QIP scenarios of interest, $S$ undergoes continuous-time dissipative dynamics due to the coupling to a (physical or engineered) Markovian environment, in which case the resulting dynamics is described by a Lindblad master equation [1, 29]. In units where $\hbar=1$, we may write

$$
\begin{aligned}
\dot{\rho}(t) & \equiv \mathcal{L}[\rho(t)] \\
& =-i[H, \rho(t)]+\sum_{k}\left(L_{k} \rho(t) L_{k}^{\dagger}-\frac{1}{2}\left\{L_{k}^{\dagger} L_{k}, \rho(t)\right\}\right),
\end{aligned}
$$

in which $\mathcal{L}$ represents the most general form for the generator of a completely-positive, trace-preserving Markov semigroup, $\left\{\mathcal{T}_{t} \equiv e^{\mathcal{L} t}, t \geq 0\right\}$, acting on $\mathfrak{D}(\mathcal{H})$. Here, $H$ is an Hermitian operator associated with the Hamiltonian of $S$, whereas the Lindblad (or noise) operators $\left\{L_{k}\right\}$ specify the non-Hamiltonian component of the generator, resulting in non-unitary irreversible dynamics. It is worth recalling that, for a given Markovian generator, the decomposition into Hamiltonian and dissipative part in Eq. (11) is not unique, in the sense that

$$
\mathcal{L}\left(H,\left\{L_{k}\right\}\right) \equiv \mathcal{L}\left(\tilde{H},\left\{\tilde{L}_{k}\right\}\right),
$$

provided that the relevant operators are redefined as follows, see e.g. Lemma 2 in [12]:

$$
\tilde{L}_{k} \equiv L_{k}+c_{k} I, \quad \tilde{H} \equiv H+\frac{i}{2} \sum_{k}\left(c_{k}^{*} L_{k}-c_{k} L_{k}^{\dagger}\right), \quad c_{k} \in \mathbb{C} .
$$


Given a dynamical evolution of the above form, locality constraints are imposed by requiring both the Hamiltonian and each Lindblad operator to have a non-trivial (non-identity) action only on certain subsets of subsystems, which may be distinguished by the geometry of the system and/or a physical coupling topology, and which we shall henceforth refer to as neighborhoods. Following [36], neighborhoods $\left\{\mathcal{N}_{j}\right\}$ can be specified in full generality as subsets of the set of indexes labeling the subsystems, that is,

$$
\mathcal{N}_{j} \subsetneq\{1, \ldots, n\}, \quad j=1, \ldots, M
$$

Once a neighborhood structure is assigned on $\mathcal{H}$, a list of reduced neighborhood states $\left\{\rho_{\mathcal{N}_{j}}\right\}$ can also be naturally associated to every state of $S$ :

$$
\rho_{\mathcal{N}_{j}} \equiv \operatorname{Tr}_{\overline{\mathcal{N}}_{j}}(\rho), \quad \rho \in \mathfrak{D}(\mathcal{H}), \quad j=1, \ldots, M,
$$

where $\operatorname{Tr}_{\overline{\mathcal{N}}_{\mathrm{j}}}$ indicates the partial trace over the tensor complement of the neighborhood $\mathcal{N}_{j}$, namely, $\mathcal{H}_{\overline{\mathcal{N}}_{j}}=$ $\bigotimes_{a \notin \mathcal{N}_{j}} \mathcal{H}_{a}$. A Lindblad master equation as in Eq. (1) will be called QL if both its Hamiltonian and each of the noise operators are QL, according to the following:

Definition $1 A$ Lindblad operator $L$ is QL if there exists a neighborhood $\mathcal{N}_{j}$ such that:

$$
L \equiv L_{\mathcal{N}_{j}} \otimes I_{\overline{\mathcal{N}}_{j}}
$$

where $L_{\mathcal{N}_{j}}$ accounts for the action of $L$ on the subsystems in $\mathcal{N}_{j}$, and $I_{\overline{\mathcal{N}}_{j}}:=\bigotimes_{a \notin \mathcal{N}_{j}} I_{a}$ is the identity on the remaining ones. Similarly, a Hamiltonian $H$ is $\mathrm{QL}$ if it admits a decomposition into a sum of $Q L$ terms:

$$
H=\sum_{j} H_{j}, \quad H_{j} \equiv H_{\mathcal{N}_{j}} \otimes I_{\overline{\mathcal{N}}_{j}} .
$$

It is immediate to verify that the QL property is well defined with respect to the freedom in the representation of the Markov generator, since the transformation in Eq. (2) preserves the quasi-locality character of $H$ and $\left\{L_{k}\right\}$. We also note that introducing locality constraints based on neighborhoods formally encompasses different specific notions that are encountered in the physical and QIP literature [1, 11, 14], where locality is typically associated with operators of fixed maximum weight $t$ (also called " $t$ body" or "t-local" interactions) and/or "distance" on a graph. For instance, when neighborhoods coincide with individual subsystems, strictly local (or "single-site") dynamics is enforced, with the corresponding propagator $\mathcal{T}_{t}$ being fully factorized relative to the multipartite structure, $\mathcal{T}_{t} \equiv \otimes_{a=1}^{n} \mathcal{T}_{a, t}$ [12]. Likewise, for $t=2$, allowing for arbitrary two-body (possibly long-range) interactions correspond to identifying neighborhoods with all possible subsystem pairs, whereas interactions between nearestneighbors sites may be accounted for by restricting the neighborhoods accordingly.

\section{B. Stability definitions and standard form for stabilization}

As mentioned, our focus in this paper is on stabilizing a desired pure state of $S$. The relevant notions of stability are given in the following:

Definition 2 Let $\rho_{d}=|\Psi\rangle\langle\Psi| \in \mathfrak{D}(\mathcal{H})$ be a pure state of $S$ evolving under Eq. (11).

(i) $\rho_{d}$ is Globally Asymptotically Stable (GAS) if for every initial condition $\rho_{0} \in \mathfrak{D}(\mathcal{H})$ we have

$$
\lim _{t \rightarrow+\infty} e^{\mathcal{L} t}\left[\rho_{0}\right]=\rho_{d}
$$

(ii) If $\mathcal{H}^{\prime} \subsetneq \mathcal{H}$ is a proper subspace of $\mathcal{H}, \rho_{d}$ is Conditionally Asymptotically Stable relative to $\mathcal{H}^{\prime}$ (or simply $\mathcal{H}^{\prime}-\mathrm{AS}$ ) if Eq. (4) holds for $\rho_{0} \in \mathfrak{D}\left(\mathcal{H}^{\prime}\right)$.

A necessary condition for a state to be GAS is that it is invariant [51], namely, that it is in the kernel of the Liouvillian, $\mathcal{L}\left(\rho_{d}\right)=0$. The following proposition, that follows from the general results of [12], provides a particularly simple way to check for invariance if $\rho_{d}$ is pure:

Proposition 1 Let the dynamics be driven by $\mathcal{L}\left(H,\left\{L_{k}\right\}\right)$ as in Eq. (11). Then a state $\rho_{d}=|\Psi\rangle\langle\Psi| \in \mathfrak{D}(\mathcal{H})$ is invariant if and only if

$$
\begin{aligned}
L_{k}|\Psi\rangle & =\ell_{k}|\Psi\rangle, \quad \ell_{k} \in \mathbb{C}, \forall k, \\
\tilde{H}|\Psi\rangle=h|\Psi\rangle, \quad \tilde{H} & =H-\frac{i}{2} \sum_{k}\left(\ell_{k}^{*} L_{k}-\ell_{k} L_{k}^{\dagger}\right), h \in \mathbb{R}(6)
\end{aligned}
$$

Proof: Let $\mathcal{H}_{d} \equiv \operatorname{span}\{|\Psi\rangle\}$ and, with respect to the natural representation induced by the partition $\mathcal{H}=$ $\mathcal{H}_{d} \oplus \mathcal{H}_{d}^{\perp}$, let operators be given in the block form

$$
X=\left[\begin{array}{l|l}
X_{d} & X_{P} \\
\hline X_{Q} & X_{R}
\end{array}\right],
$$

where the top-left block is one-dimensional. Given Corollary 1 in [12], it follows that $\rho_{d}$ is invariant if and only if $L_{k}|\Psi\rangle=l_{k, d}|\Psi\rangle \equiv l_{k}|\Psi\rangle$ for all $k$, and the entries in the $P$-block obey $i H_{P}-(1 / 2) \sum_{k} \ell_{k}^{*} L_{P, k}=0$. Clearly, if $\ell_{k} \equiv 0$ in Eq. (5), then it follows that $H_{P}=0=H_{Q}$ by Hermiticity. Therefore, $H \equiv \tilde{H}$ is block-diagonal, with $H|\Psi\rangle=h_{d}|\Psi\rangle \equiv h_{d}|\Psi\rangle$, as stated in (6). If $\ell_{k} \neq 0$ for some $k$, we can use Eq. (2) to redefine $\tilde{L}_{k} \equiv L_{k}-\ell_{k} I$ and, accordingly, $\tilde{H}$ as in (6). Since now $\tilde{\ell}_{k} \equiv 0, \tilde{H}$ is, again, block-diagonal, with $|\Psi\rangle$ being an eigenstate with eigenvalue $\tilde{h}_{d} \equiv h$, as stated.

Thanks to the above result, it is always possible to express a Markovian generator that asymptotically stabilizes a pure state in a standard form, in which the desired target state $|\Psi\rangle$ is annihilated by all the noise operators (thus being a "dark state" in quantum-optics language 11]) and is an eigenstate of the corresponding Hamiltonian. That is: 
Corollary 1 If a generator $\mathcal{L}\left(H,\left\{L_{k}\right\}\right)$ makes $\rho_{d}=$ $|\Psi\rangle\langle\Psi| \in \mathfrak{D}(\mathcal{H})$ GAS, then the same generator can be represented in a standard form $\mathcal{L}\left(\tilde{H},\left\{\tilde{L}_{k}\right\}\right)$, in such a way that $\tilde{H}|\Psi\rangle=h|\Psi\rangle$ and $\tilde{L}_{k}|\Psi\rangle=0$, for all $k$.

\section{Quasi-local stabilization settings}

In general, a QL stabilization problem will entail the specification of the desired target state and the relevant QL constraints, along with a description of the target dynamical model and control parameters. While, as mentioned, we shall focus here on pure target states, different stabilization settings may be envisioned depending on the nature of the available control resources and initialization capabilities, and on the existence of non-trivial Markovian dynamics in the absence of control, hereby referred to as drift in the standard control-theory terminology and characterized by a Liouvillian generator $\mathcal{L}_{0}$. Specifically, let us consider a class of dynamical models as in Eq. (1), with the corresponding generator $\mathcal{L} \equiv \mathcal{L}\left(H,\left\{L_{k}\right\}\right)$ taken to be in standard form henceforth, and

$$
\dot{\rho}(t)=\mathcal{L}(\rho(t)) \equiv\left[\mathcal{L}_{0}+\mathcal{L}_{c}\right](\rho(t)) .
$$

Here, $\mathcal{L}_{0}\left(H_{0},\left\{L_{k}^{0}\right\}\right)$ and $\mathcal{L}_{c}\left(H_{c},\left\{D_{k}\right\}\right)$ are associated to the drift and control dynamics, respectively, and $H=$ $H_{0}+H_{c},\left\{L_{k}\right\}=\left\{L_{k}^{0}, D_{k}\right\}$. Hamiltonian control is introduced by the time-independent $H_{c}$, whereas each of the Lindblad operators $D_{k}$ represent a different incoherent control knob on $S$. We can then introduce the following definitions.

Definition 3 A pure state $\rho_{d}=|\Psi\rangle\langle\Psi| \in \mathfrak{D}(\mathcal{H})$ is:

(i) Quasi-Locally Stabilizable (QLS) if there exist a $Q L$ Hamiltonian $H_{c}$ and $Q L$ noise operators $\left\{D_{k}\right\}_{k=1, \ldots, K}$ in standard form, such that $\rho_{d}$ is GAS for the controlled Lindblad evolution in Eq. (7).

(ii) Dissipatively Quasi-Locally Stabilizable (DQLS) if it is $Q L S$ with $H_{c} \equiv 0$ and $Q L$ noise operators $\left\{D_{k}\right\}_{k=1, \ldots, K}$ in standard form.

(iii) Conditionally DQLS with respect to a subspace $\mathcal{H}^{\prime} \subsetneq \mathcal{H}$ (or simply $\mathcal{H}^{\prime}$-DQLS), if there exist $Q L$ noise operators $\left\{D_{k}\right\}_{k=1, \ldots, K}$ in standard form, such that $\rho_{d}$ is conditionally $A S$ relative to $\mathcal{H}^{\prime}$ for the dissipatively controlled Lindblad evolution in Eq. (7).

Two features follow from the global stability properties given in Definition 2, while exact QL preparation cannot be achieved in finite time, convergence happens exponentially fast in time 35]. Furthermore, if the target state is stabilizable, according to the appropriate definition, the stabilizing dynamics is robust with respect to the initial state 12] thus, equivalently, the control parameters are all-to-one [18].

Clearly, the QLS setting includes both purely dissipative and conditional stabilization as special instances where either (or both) the admissible control actions and initial states are restricted, respectively. Introducing a separate DQLS notion is motivated by the fact that it allows for a simpler mathematical treatment (see Sec. IID) while being adequate for important classes of entangled pure states and, from a practical standpoint, potentially simpler to implement. For states which are not DQLS, QLS may or may not be achievable. Even in the latter case, conditional stabilization may offer a practical method of choice in situations where a two-step "switched" dynamics, that first initializes $S$ to the intended subspace, and from there enacts dissipative stabilization, is preferable over identifying and implementing a combined Hamiltonian-dissipative control action - for instance thanks to the presence of a conserved quantity 5]. While a number of illustrative examples will be discussed in Sec. VI ensuring that the required number $K$ of noise operators scales favorably with the complexity of the neighborhood structure is crucial in practice. The situation is straightforward for the DQLS setting, since it follows directly from Theorem 1 below that a single noise operator per neighborhood always suffices $(K=M)$. In general, the randomized algorithms that we will provide to achieve stabilization in case (i) and (ii) will also output, when successful, a noise operator per neighborhood, as we shall see.

We begin by presenting two preliminary results that directly generalize their counterparts in Ref. [36] for the DQLS case. First, let $U=\bigotimes_{a=1}^{n} U_{a}$ be an arbitrary local unitary (LU) transformation. Then it is straightforward to see that the above stabilization notions are invariant under arbitrary LU transformations of the target state, as desirable given that entanglement properties are themselves preserved under LUs. The following Proposition may be established through the same steps used in Lemma 2.2 and Proposition 2.3 in [36]:

Proposition 2 If $\rho_{d}$ is $Q L S$ and $U$ is any $L U$, then $\rho_{d}^{\prime}=$ $U \rho_{d} U^{\dagger}$ is also $Q L S$. If $\rho_{d}$ is $\mathcal{H}^{\prime}-D Q L S$ and $U$ is any $L U$ that leaves $\mathcal{H}^{\prime}$ invariant, then $\rho_{d}^{\prime}=U \rho_{d} U^{\dagger}$ is also $\mathcal{H}^{\prime}$ $D Q L S$.

A second basic yet useful result regards the structure of the operators $\left\{D_{k}\right\}$ : the Lemma that follows shows that the support of a QLS state must still be, as in the DQLS case, contained in the kernel of the noise operators written in standard form:

Lemma 1 Assume that the generator associated to $Q L$ $\left\{H, D_{k}\right\}$ leaves $\rho_{d}=|\Psi\rangle\langle\Psi|$ invariant. Then, for each $k$, we have $\operatorname{supp}\left(\rho_{\mathcal{N}_{\mathrm{k}}}\right) \subseteq \operatorname{ker}\left(\mathrm{D}_{\mathcal{N}_{\mathrm{k}}}\right)$.

Proof: If the state is invariant, by Proposition 1, $|\Psi\rangle$ must be in the kernel of each $D_{k}$. Thus, with respect to the decomposition $\mathcal{H}=\mathcal{H}_{d} \oplus \mathcal{H}_{d}^{\perp}$, with $\mathcal{H}_{d}=\operatorname{span}\{|\Psi\rangle\}$ as before, every $D_{k}$ must be of block form [13]:

$$
D_{k}=\left[\begin{array}{cc}
0 & D_{P, k} \\
0 & D_{R, k}
\end{array}\right],
$$


which immediately implies $D_{k} \rho_{d} D_{k}^{\dagger}=0$. It then follows that $\operatorname{Tr}_{\overline{\mathcal{N}}_{\mathrm{k}}}\left(D_{k} \rho_{d} D_{k}^{\dagger}\right)=0$, therefore $\operatorname{Tr}_{\overline{\mathcal{N}}_{\mathrm{k}}}\left(D_{\mathcal{N}_{k}} \otimes\right.$ $\left.I_{\overline{\mathcal{N}}_{k}} \rho_{d} D_{\mathcal{N}_{k}}^{\dagger} \otimes I_{\overline{\mathcal{N}}_{k}}\right)=0$. Thus, it also follows that $D_{\mathcal{N}_{k}} \rho_{\mathcal{N}_{k}} D_{\mathcal{N}_{k}}^{\dagger}=0$. If we consider the spectral decomposition $\rho_{\mathcal{N}_{k}} \equiv \sum_{j} q_{j}\left|\phi_{j}\right\rangle\left\langle\phi_{j}\right|$, with $q_{j}>0$, the latter implies that, for each $j, \tilde{D}_{\mathcal{N}_{k}}\left|\phi_{j}\right\rangle\left\langle\phi_{j}\right| \tilde{D}_{\mathcal{N}_{k}}^{\dagger}=0$. Thus, it must be $\operatorname{supp}\left(\rho_{\mathcal{N}_{\mathrm{k}}}\right) \subseteq \operatorname{ker}\left(\tilde{\mathrm{D}}_{\mathcal{N}_{\mathrm{k}}}\right)$, as stated.

For added clarity and notational simplicity, we shall from now on assume that the dynamics of the system is drift-less, that is, $\mathcal{L}_{0} \equiv 0$ in Eq. (77). After briefly recalling the main results on DQLS, we will present our new results on Hamiltonian-assisted and conditional QL stabilization under the simplyfing drift-less assumption, in Sec. III and Sec. IV, respectively. We will then allow for drift dynamics and explicitly address its role in Sec. V]

\section{Prior results for quasi-local dissipative stabilization}

A characterization of DQLS states, leading to a simple linear-algebraic algorithm to test whether a given pure state is DQLS, may be obtained based on the properties of the reduced states on the neighborhoods [52], as defined in Eq. (3). Let

$$
\mathcal{H}_{k}^{\circ} \equiv \operatorname{supp}\left(\rho_{\mathcal{N}_{k}} \otimes I_{\overline{\mathcal{N}}_{k}}\right), \quad \mathcal{H}_{0}=\bigcap_{k} \mathcal{H}_{k}^{\circ} .
$$

We thus have the following:

Theorem 1 [36] A pure state $\rho_{d}=|\Psi\rangle\langle\Psi|$ is DQLS if and only if

$$
\operatorname{supp}\left(\rho_{d}\right)=\mathcal{H}_{0} .
$$

The proof of this Theorem includes an explicit construction of a choice of stabilizing noise operators, with their general block-structure highlighted in Lemma 1 . It also points to natural connections with the formalism of parent Hamiltonians and the concept of frustration from many-body physics. Let $H=\sum_{k} H_{k}$, with $H_{k}=H_{\mathcal{N}_{k}} \otimes I_{\overline{\mathcal{N}}_{k}}$ and, as before, the index $k$ refers to the $k$ th neighborhood. A QL Hamiltonian $H$ is said to be a parent Hamiltonian for $|\Psi\rangle$, if $|\Psi\rangle$ is an exact ground state for $H$ [30]. In addition, $|\Psi\rangle$ is said to be frustration-free if it is the exact ground state of each Hamiltonian $H_{k}$ separately, that is, $\left\langle\Psi\left|H_{k}\right| \Psi\right\rangle=\min \lambda\left(H_{k}\right), \forall k$, where $\lambda(\cdot)$ denotes the spectrum of a matrix. Remarkably, it is known that for a large class of product entangled-pair states (PEPSs) or, in one spatial dimension, matrix product states (MPSs), a frustration-free parent Hamiltonian may be constructed [53], which has the desired state as its unique ground state [32 34]. The QL properties of this Hamiltonian are determined by the (minimum) "bond dimension" of the corresponding PEPS or MPS representation. Suppose that a pure state is the unique ground state of a frustration-free parent Hamiltonian. Then the QL structure of $H$ may be naturally used to derive a stabilizing semigroup [11]. It is easy to show that this condition is also necessary, leading to the following:

Corollary $2[36]$ A state $\rho_{d}=|\Psi\rangle\langle\Psi|$ is DQLS if and only if it is the unique ground state of a frustration-free parent Hamiltonian.

Despite the formal points of contact, it is crucial to remark that in MPS-based stabilization approaches 11. the relevant QL notion is intrinsically state-dependent, whereas it is taken to be a fixed problem input in our control-motivated approach. From a practical standpoint, the DQLS class includes important representative sets of entangled states - most notably, all stabilizer and graph states [11, 36], relative to the natural choice of neighborhoods associated with connected nodes on the graph. Still, paradigmatic examples of genuinely multipartite entangled states such as GHZ and W states can be easily seen to fail the DQLS test in Eq. (9), except in "fully connected" (for example, "star") coupling topologies where the QL constraint becomes effectively trivial. While we defer to [36] and Sec. VI for further discussion, we shall proceed to separately formalize and analyze the general QLS and the conditional DQLS scenarios next.

\section{QUASI-LOCAL STABILIZATION WITH CONSTANT DISSIPATIVE AND HAMILTONIAN CONTROL}

\section{A. Necessary conditions}

Assume that the target state $\rho_{d}=|\Psi\rangle\langle\Psi|$ is not DQLS, and let $\mathcal{H}_{d}=\operatorname{span}\{|\Psi\rangle\}$ as before, corresponding to the support of the desired state. In terms of $\mathcal{H}_{0}$, defined as in Eq. (8), this translates to:

$$
\operatorname{dim}\left(\mathcal{H}_{0}\right)=\operatorname{dim}\left(\bigcap_{k} \mathcal{H}_{k}^{\circ}\right) \equiv d_{0} \geq 2 .
$$

Our first result is the following necessary condition for QLS (recall that $H \equiv H_{c}$ in the drift-less scenario we consider for now):

Proposition $3 \rho_{d}$ is $Q L S$ but not DQLS only if $\mathcal{H}_{d}$ is an invariant subspace for the Hamiltonian $H_{c}$ and no other invariant subspace is contained in (or equal to) $\mathcal{H}_{0}$. In particular, one can choose $H_{c}$ so that $H_{c}|\Psi\rangle=0$.

Proof: If there were another invariant subspace for the Hamiltonian with support in $\mathcal{H}_{0}$, the latter would be, by definition of $\mathcal{H}_{0}$, also in the kernel of each $D_{k}$, and hence it would be invariant. Invariant subspaces always contain at least an invariant state. It then follows that $\rho_{d}$ could not be GAS. If $H_{c}|\Psi\rangle=\lambda|\Psi\rangle, \lambda \neq 0$, we can 
always choose $H_{c}^{\prime}=H_{c}-\lambda I$ instead, which is also QL if $H_{c}$ was.

When such a stabilizing Hamiltonian $H_{c}$ exists, one must look for noise operators $\left\{D_{k}\right\}$ such that $\mathcal{H}_{d}$ is the only invariant subspace for the whole generator $\mathcal{L}_{c}\left(H_{c},\left\{D_{k}\right\}\right)$, and thus makes $\rho_{d}$ GAS. Lemma 1 suggests that the most effective choice of noise operators can stabilize $\mathcal{H}_{0}$, but do no better than that. In order to specify what the action of a stabilizing Hamiltonian would be, it is convenient to pick an orthonormal basis for $\mathcal{H}_{0}$, which includes the target state:

$$
\mathcal{H}_{0}=\operatorname{span}\left\{|\Psi\rangle,\left|\Phi_{1}\right\rangle, \ldots,\left|\Phi_{r}\right\rangle\right\}, \quad r=d_{0}-1 .
$$

One would hope that $H_{c}\left|\Phi_{j}\right\rangle \notin \mathcal{H}_{0}$ for each $j$. However, fulfilling these conditions is clearly not necessary, and in fact it need not be possible given the QL constraint. However, in the simplest case of $d_{0}=2$, the above idea leads to a specialized formulation of Proposition 3 .

Corollary $3 \rho_{d}$ is $Q L S$ but not DQLS, with $\mathcal{H}_{0}=$ span $\left\{|\Psi\rangle,\left|\Phi_{1}\right\rangle\right\}$, only if there exists a $Q L$ Hamiltonian $H_{c}$ such that

$$
H_{c}|\Psi\rangle=0, \quad H_{c}\left|\Phi_{1}\right\rangle \notin \mathcal{H}_{0} .
$$

Recall that in the DQLS context (Corollary 2), a QL parent Hamiltonian was naturally associated to the action of the noise operators, in such a way that $\mathcal{H}_{0}$ was the common ground eigenspace of all the QL components, that is, with no frustration involved. In contrast, the necessary conditions provided above clearly show that an effective control Hamiltonian $H_{c}$ cannot be frustration-free with respect to $\mathcal{H}_{0}$, since it must destabilize some part of $\mathcal{H}_{0}$ in order to attain GAS of the target state.

In Sec. VI we will employ Corollary 3 to construct a stabilizing Hamiltonian for both GHZ and W states which, as noted earlier, are never DQLS under non-trivial QL constraints.

\section{B. Randomized construction of stabilizing Hamiltonian and dissipators}

Even if we succeed in finding a Hamiltonian $H_{c}$ that satisfies Proposition 3 (or Corollary 3), a procedure for determining the existence and, possibly, the actual form of the stabilizing $D_{k}$ is required in order to establish QLS. As we will illustrate with an example in Sec. VIB not all choices of $\left\{D_{k}\right\}$ satisfying Lemma 1 are effective. In fact, care is needed in ensuring that the interplay between Hamiltonian and dissipative control introduces enough "mixing" and does not allow for other invariant sets to exist.

With that in mind, we shall invoke a randomized design approach that expands our earlier use of randomization in [35] to both the Hamiltonian and the dissipative component, and prove that a generic choice of noise operators which satisfy Lemma 1 and stabilize $\mathcal{H}_{0}$ will suffice to achieve QLS. Specifically, assume that we represent the desired stabilizing operators $H_{c}=\sum_{k} H_{k}$, and $\left\{D_{k}\right\}$ in parametric form:

$$
H_{k}=\sum_{j} \alpha_{j k} \sigma_{j k}, \quad D_{k}=\sum_{j} \beta_{j k} \sigma_{j k},
$$

where $\alpha_{j k}, \beta_{j k} \in \mathbb{R}$ are chosen at random with uniform distribution in an interval $\mathcal{I} \equiv[-\gamma, \gamma]$ of the real axis, and $\left\{\sigma_{j k}\right\}$ is a basis 54 for the space of QL operators on the $k$ th neighborhood $\mathcal{N}_{k}$. Our main result is then contained in the following:

Theorem 2 If there exists a choice of $\alpha_{j k}, \beta_{j k} \in \mathcal{I}$ that makes $\rho_{d} Q L S$, then almost any choice of $\alpha_{j k}, \beta_{j k} \in I$, that makes $\rho_{d}$ invariant, makes it $Q L S$ as well.

While the proof of the above Theorem is rather technical (see Appendix VIII), the meaning is clear: imposing invariance requires "fine-tuning" of the parameters, however once invariance is ensured, if GAS is possible, then it comes almost always for free. An algorithm for constructing a stabilizing QL Hamiltonian and achieve QLS may then be provided as follows.

Step 1: Imposing quasi-locality of $\left\{H_{c}\right\}$. Pick a product operator basis for the $d^{2}$-dimensional space of linear operators $\mathfrak{B}(\mathcal{H})$, say, $\left\{\sigma_{i_{1}} \otimes \ldots \otimes\right.$ $\left.\sigma_{i_{n}} \mid i_{a}=1, \ldots, d_{a}^{2}\right\}$. Let $H_{c}=\sum_{k} H_{k}$, and associate each $H_{k}$ and $D_{k}$ to a vector $\vec{h}_{k}$ and $\vec{d}_{k}$, respectively. Let $\hat{D}=\left[\vec{d}_{1}|\cdots| \vec{d}_{n}\right]$ be the matrix of the coefficient of the noise operators relative to the above product basis, and $\hat{B}_{k}$ the orthogonal projection onto the subspace generated by the basis elements that are QL with respect to $\mathcal{N}_{k}$, with $\hat{B}_{k}^{\perp}=I-\hat{B}_{k}$. We must then require, for each $k$ :

$$
\hat{B}_{k}^{\perp} \vec{h}_{k}=0, \quad \hat{B}_{k}^{\perp} \vec{d}_{k}=0 .
$$

Step 2: Ensuring invariance of $\mathcal{H}_{0}$. Impose the linear constraints on the QL noise operators and on the Hamiltonian, namely $D_{k}|\psi\rangle=0, H_{c}|\psi\rangle=0$ for all $k$. If $\hat{P}_{0}$ is the matrix representation of the linear (super)-operator $P_{0}(X)=X|\psi\rangle\langle\psi|$ with respect to the the chosen basis, this is equivalent to require that for each $k$,

$$
\hat{P}_{0} \vec{h}=0, \quad \hat{P}_{0} \vec{d}_{k}=0 .
$$

These two steps translate, for each $k$, in the following homogeneous systems of linear equations:

$$
\left[\begin{array}{c}
\hat{B}_{k}^{\perp} \\
\hat{P}_{0}
\end{array}\right] \vec{d}_{k}=0, \quad\left[\begin{array}{c}
\hat{B}_{k}^{\perp} \\
\hat{P}_{0}
\end{array}\right] \vec{h}_{k}=0 .
$$

Step 3: Enforcing convergence by randomization. If the constraints above allow for non-trivial solutions (and hence a subspace of solutions since the system is homogenous), choose the free variables uniformly at random within a finite interval $[-\gamma, \gamma] \subset \mathbb{R}$. 
We thus have the following immediate corollary of Theorem 2 ,

Corollary 4 If $\rho_{d}$ is $Q L S$, the $Q L$ generator $\mathcal{L}_{c}\left(H_{c},\left\{D_{k}\right\}\right)$ constructed in Steps 1-3 makes it $G A S$ with probability one. If $\rho_{d}$ is not $Q L S$, then it is not stabilized by the constructed Hamiltonian $H_{c}$ and noise operators $\left\{D_{k}\right\}$.

Since checking whether a state is the unique equilibrium of some Lindblad dynamics is straightforward (e.g., by checking that the superoperator form of the corresponding generator has a unique unit eigenvalue), the above algorithm can be used as an explicit test for QLS.

\section{CONDITIONAL QUASI-LOCAL DISSIPATIVE STABILIZATION}

\section{A. Characterization of conditional stability}

When a state is not DQLS and Hamiltonian control is not viable, we can return to a purely dissipative control setting and analyze our second proposed stabilization strategy, namely to restrict the initial state to a given initial subspace of $\mathcal{H}$. With $\mathcal{H}_{d}=\operatorname{span}\{|\Psi\rangle\}$ and $\mathcal{H}_{0}$ given in Eq. (8) as before, let us additionally define the following subspaces:

$$
\mathcal{H}_{w}=\mathcal{H}_{0} \ominus \mathcal{H}_{d}, \quad \mathcal{H}_{r}=\mathcal{H} \ominus \mathcal{H}_{0} .
$$

By construction, $\mathcal{H}_{w}$ corresponds to the portion of the subspace stabilizable by purely dissipative means that is orthogonal to the target. Assume that the dynamics is given by Eq. (7), with $\mathcal{L} \equiv 0$, associated to QL noise operators $\left\{D_{k}\right\}$ that make $\mathfrak{D}\left(\mathcal{H}_{0}\right)$ GAS. We aim to characterize which choice $(\mathrm{s})$ of $\mathcal{H}^{\prime}$ can make $\rho_{d}$ conditionally DQLS (with the obvious requirement that $\mathcal{H}^{\prime} \supsetneq \mathcal{H}_{d}$ ). A first necessary condition is provided by the following Lemma:

\section{Lemma $2 \rho_{d}$ can be $\mathcal{H}^{\prime}-D Q L S$ only if}

$$
\mathcal{H}^{\prime} \subseteq \mathcal{H} \ominus \mathcal{H}_{w}=\mathcal{H}_{d} \oplus\left[\mathcal{H} \ominus \mathcal{H}_{0}\right]
$$

Proof: Since $\mathfrak{D}\left(\mathcal{H}_{w}\right) \subsetneq \mathfrak{D}\left(\mathcal{H}_{0}\right)$, and by hypothesis $\mathfrak{D}\left(\mathcal{H}_{0}\right)$ is invariant under the Lindblad dynamics $\mathcal{L}_{D}$ induced by the $\left\{D_{k}\right\}$, then $\mathfrak{D}\left(\mathcal{H}_{w}\right)$ is invariant as well. If $\mathcal{H}^{\prime} \subseteq \mathcal{H} \ominus \mathcal{H}_{w}$ did not hold, then there would be a $\rho \in \mathfrak{D}\left(\mathcal{H}^{\prime}\right)$ such that $\operatorname{Tr}\left(\rho \Pi_{\mathcal{H}_{w}}\right)>0$, with $\Pi_{\mathcal{H}_{w}}$ denoting the projector onto $\mathcal{H}_{w}$. Define $p_{w}=\operatorname{Tr}\left(\Pi_{\mathcal{H}_{w}} \rho\right)>0$, $\rho_{w}=p_{w}^{-1} \Pi_{\mathcal{H}_{w}} \rho \Pi_{\mathcal{H}_{w}} \in \mathfrak{D}\left(\mathcal{H}_{w}\right)$, and write $\rho=p_{w} \rho_{w}+\Delta \rho$, for some Hermitian but not necessarily positive operator $\Delta \rho$. Since the dynamics is linear and $\rho_{w}$ invariant, $\operatorname{Tr}\left(\rho \Pi_{\mathcal{H}_{w}}\right)$ cannot decrease along the dynamical flow [12], therefore we may write

$\lim _{t \rightarrow \infty} \operatorname{Tr}\left(e^{\mathcal{L}_{D} t}[\rho] \Pi_{\mathcal{H}_{w}}\right)=\operatorname{Tr}\left(e^{\mathcal{L}_{D} t}[\Delta \rho]\right)+p_{w} \operatorname{Tr}\left(\rho_{w}\right) \geq p_{w}>0$.
Hence $\rho \in \mathfrak{D}\left(\mathcal{H}^{\prime}\right)$ would not converge to $\rho_{d}$.

The following "enlargement lemma" indicates that we can construct larger stabilizing subspaces out of smaller ones, both weakening our constraints and gaining a key property: invariance. Formally:

Lemma 3 If $\rho_{d}$ is $\mathcal{H}^{\prime \prime}-D Q L S$, it is also $\mathcal{H}^{\prime}-D Q L S$ for some $\mathcal{H}^{\prime}$ such that $\mathcal{H}^{\prime \prime} \subset \mathcal{H}^{\prime}$ and $\mathfrak{D}\left(\mathcal{H}^{\prime}\right)$ is an invariant set.

Proof: In order to make $\mathfrak{D}\left(\mathcal{H}^{\prime}\right)$ invariant, it suffices to combine the supports of all trajectories originated in $\mathfrak{D}\left(\mathcal{H}^{\prime \prime}\right)$. Given two subspaces $\mathcal{H}_{1,2}$, let us denote by $\mathcal{H}_{1} \vee \mathcal{H}_{2}$, the smallest subspace that contains both of them. Define

$$
\mathcal{H}_{\rho_{0}}=\bigvee_{t \geq 0} \operatorname{supp}\left(\mathrm{e}^{\mathcal{L}_{\mathrm{D}}^{\mathrm{t}}}\left[\rho_{0}\right]\right), \quad \mathcal{H}^{\prime}=\bigvee_{\rho_{0} \in \mathfrak{D}\left(\mathcal{H}^{\prime \prime}\right)} \mathcal{H}_{\rho_{0}} .
$$

By assumption, $\mathcal{H}_{d} \subsetneq \mathcal{H}^{\prime}, \mathcal{H}^{\prime \prime} \subsetneq \mathcal{H}^{\prime}$ and $\mathfrak{D}\left(\mathcal{H}^{\prime}\right)$ is invariant. Lastly, all trajectories originated in $\mathfrak{D}\left(\mathcal{H}^{\prime}\right)$ can be obtained from trajectories originated in $\mathfrak{D}\left(\mathcal{H}^{\prime \prime}\right)$, which by hypothesis all converge to $\rho_{d}$, establishing the desired result.

Motivated by the above, we now restrict to subspaces $\mathcal{H}^{\prime}$ such that $\mathfrak{D}\left(\mathcal{H}^{\prime}\right)$ is invariant. With a slight abuse in terminology, we shall simply refer to subspaces obeying such a property as invariant. We are then ready to state our main result:

Theorem 3 Assume that the $Q L$ dissipative dynamics generated by the $\left\{D_{k}\right\}$ makes $\mathfrak{D}\left(\mathcal{H}_{0}\right) G A S$. If $\mathcal{H}^{\prime}$ satisfies the necessary condition given in Eq. (13) and is invariant for all $\left\{D_{k}\right\}$, then $\rho_{d}$ is $\mathcal{H}^{\prime}-D Q L S$.

Proof: Since $\mathcal{H}^{\prime}$ is invariant for all $\left\{D_{k}\right\}$, it follows that $\mathfrak{D}\left(\mathcal{H}^{\prime}\right)$ is a positive invariant set. By LaSalle theorem [31], this means that all the trajectories starting in $\mathfrak{D}\left(\mathcal{H}^{\prime}\right)$ converge to its largest invariant set. On the other hand, by hypothesis, $\mathfrak{D}\left(\mathcal{H}_{0}\right)$ is GAS, so the largest invariant set must be contained in $\mathfrak{D}\left(\mathcal{H}_{0}\right) \cap \mathfrak{D}\left(\mathcal{H}^{\prime}\right)$. Since by (13) we know that $\mathcal{H}_{w} \perp \mathcal{H}^{\prime}$, it follows that

$$
\mathfrak{D}\left(\mathcal{H}_{0}\right) \cap \mathfrak{D}\left(\mathcal{H}^{\prime}\right)=\mathfrak{D}\left(\mathcal{H}_{d}\right)=\rho_{d},
$$

as desired.

A further advantage of considering an invariant subspace $\mathcal{H}^{\prime}$ is highlighted in the following corollary: if initialization in $\mathcal{H}^{\prime}$ is faulty, the error on the asymptotic result remains upper-bounded by the preparation error:

Corollary 5 Let $\rho_{d}$ be $\mathcal{H}^{\prime}-D Q L S$, with $\mathcal{H}^{\prime}$ invariant under the dissipative dynamics. If $\rho$ is such that

$$
1-\operatorname{Tr}\left(\Pi_{\mathcal{H}^{\prime}} \rho\right) \equiv \varepsilon>0,
$$

then we have:

$$
\lim _{t \rightarrow \infty} \operatorname{Tr}\left(\mathrm{e}^{\mathcal{L}_{\mathrm{D}}^{\mathrm{t}}}[\rho] \Pi_{\mathcal{H}^{\prime}}\right)<\varepsilon .
$$


Proof: Since $\mathcal{H}^{\prime}$ is invariant, $\operatorname{Tr}\left(\Pi_{\mathcal{H}^{\prime}} \rho\right)$ is non decreasing along the trajectories $e^{\mathcal{L} t} \rho$, for $t \geq 0$, see [12].

While it seems hard to devise a fully general strategy for finding good choices of $\mathcal{H}^{\prime}$ and associated QL dissipators so that $\rho_{d}$ is $\mathcal{H}^{\prime}$-DQLS, constructive results may be obtained if the problem is further constrained. In particular, note that from a stabilization point of view, two subspace decompositions play a specially important role:

(i) one associated with the initial $(t=0)$ state-space structure, $\mathcal{H}=\mathcal{H}^{\prime} \oplus \mathcal{H}^{\prime \perp}$, with $\mathcal{H}^{\prime}$ containing the states to be attracted toward $\rho_{d}$;

(ii) one associated with the final $(t \rightarrow \infty)$ state-space structure, $\mathcal{H}_{0}=\mathcal{H}_{d} \oplus \mathcal{H}_{w}$, with $\mathcal{H}_{w}$ containing the unwanted states toward which dissipative stabilization occurs.

In a way, the two classes of conditional stabilization problems we solve can be seen to arise by imposing some natural constraints on $\mathcal{H}^{\prime \perp}$ and $\mathcal{H}_{w}$, in case (i) and (ii), respectively. Either way, it is worth remarking that any state in $\mathfrak{D}\left(\mathcal{H}^{\prime}\right)$ is asymptotically converging to $\rho_{d}$ : if control capabilities are enough to prepare a subset or even a single state in this set, dissipative QL preparation can be achieved. We begin by addressing case (ii), which is directly motivated by $\mathrm{W}$ states and is technically simpler.

\section{B. Conditional stabilization under constraints on the final attractive set}

In order to formulate and interpret the required property that $\mathcal{H}_{w}$ must obey to allow for $\mathcal{H}^{\prime}$ and associated QL dissipators to be systematically constructed, some additional definitions are needed. Let

$$
\begin{gathered}
\mathcal{H}_{\mathcal{N}_{k}}^{\circ}=\operatorname{supp}\left(\rho_{\mathcal{N}_{\mathrm{k}}}\right), \quad \mathcal{H}_{\mathcal{N}_{\mathrm{k}}}^{\mathrm{r}}=\mathcal{H}_{\mathcal{N}_{\mathrm{k}}} \ominus \mathcal{H}_{\mathcal{N}_{\mathrm{k}}}^{\circ}, \\
\mathcal{H}_{\mathcal{N}_{k}}^{w}=\operatorname{supp}\left(\operatorname{Tr}_{\overline{\mathcal{N}}_{\mathrm{k}}}\left(\Pi_{\mathcal{H}_{\mathrm{w}}}\right)\right)
\end{gathered}
$$

where as before $\Pi_{\mathcal{H}_{w}}$ is the orthogonal projector on $\mathcal{H}_{w}$. By construction, $\mathcal{H}_{w} \subseteq \mathcal{H}_{0}$ and $\mathcal{H}_{\mathcal{N}_{k}}^{w} \subseteq \mathcal{H}_{\mathcal{N}_{k}}^{\circ}$. As we shall establish, the key property that $\mathcal{H}_{w}$ must obey is the following strict inclusion for each neighborhood:

$$
\mathcal{H}_{\mathcal{N}_{k}}^{w} \subsetneq \mathcal{H}_{\mathcal{N}_{k}}^{\circ}
$$

Some intuition on the above requirement may be built as follows. We know that $\mathcal{H}_{\mathcal{N}_{k}}$ must be in the kernel of all the noise operators $D_{k}$, and hence the dynamics in this subspace is trivial. We thus would like to be able to distinguish $\mathcal{H}_{d}$ from $\mathcal{H}_{w}$, and "push" the evolution towards the former but not the latter. If Eq. (14) is obeyed, we know that if a pure state is in $\mathcal{H}_{0}$ but has no $Q L$ support on any of the $\mathcal{H}_{\mathcal{N}_{k}}^{w}$, then it must be in $\mathcal{H}_{d}$ and hence in $\rho_{d}$. Formalizing this intution provides us with a way to construct a suitable subspace $\mathcal{H}^{\prime}$ and utilize Theorem 3 to prove convergence.
Specifically, our candidate subspace for conditional invariance is defined as follows:

$$
\mathcal{H}^{\prime} \equiv \mathcal{H} \ominus \bigcap_{k} \tilde{\mathcal{H}}_{k}, \quad \tilde{\mathcal{H}}_{k}=\mathcal{H}_{\mathcal{N}_{k}}^{w} \otimes \mathcal{H}_{\overline{\mathcal{N}}_{k}} .
$$

By Lemma 1 and the definition of $\mathcal{H}_{\mathcal{N}_{k}}^{w}, \mathcal{H}_{w} \subseteq \bigcap_{k} \tilde{\mathcal{H}}_{k}$. Hence, $\mathcal{H}^{\prime}$ obeys the necessary condition established in Eq. (13):

$$
\mathcal{H}^{\prime} \subseteq \mathcal{H} \ominus \mathcal{H}_{w}
$$

We now need to construct noise operators $D_{k}=D_{\mathcal{N}_{k}} \otimes$ $I_{\overline{\mathcal{N}}_{k}}$, such that $\mathcal{H}_{0}$ and $\mathcal{H}^{\prime}$ are invariant. To this aim, define $\mathcal{H}_{\mathcal{N}_{k}}^{t} \equiv \mathcal{H}_{\mathcal{N}_{k}}^{\circ} \ominus \mathcal{H}_{\mathcal{N}_{k}}^{w}$, which by Eq. (14) is not empty, and consider $D_{\mathcal{N}_{k}}$ with the following block structure, with respect to the decomposition of the QL space $\mathcal{H}_{\mathcal{N}_{k}}=\mathcal{H}_{\mathcal{N}_{k}}^{w} \oplus \mathcal{H}_{\mathcal{N}_{k}}^{t} \oplus \mathcal{H}_{\mathcal{N}_{k}}^{r}$ :

$$
D_{\mathcal{N}_{k}}=\left[\begin{array}{ccc}
0 & 0 & 0 \\
0 & 0 & D_{P, \mathcal{N}_{k}} \\
0 & 0 & D_{R, \mathcal{N}_{k}}
\end{array}\right]
$$

The above structure can be exploited, e.g. via a choice of $D_{P, \mathcal{N}_{k}}, D_{R, \mathcal{N}_{k}}$ of "ladder form" [12], to render each of the $\mathcal{H}_{\mathcal{N}_{k}}^{\circ}$ (hence $\mathcal{H}_{0}$ ) GAS. We hereby consider this or an equivalent choice, so that $\mathcal{H}_{0}$ is made GAS with $\mathrm{QL}$ operators. In addition to this, the block structure in Eq. (16) ensures the required invariance property of $\mathcal{H}^{\prime}$ :

Proposition $4 A$ choice of $D_{k}$ satisfying Eq. (16) ensures that $\mathcal{H}^{\prime}=\mathcal{H} \ominus \bigcap_{k} \tilde{\mathcal{H}}_{k}$ is invariant.

Proof: Given the matrix structure, we have:

$$
\begin{aligned}
& D_{k} \mathcal{H}^{\prime} \subseteq D_{k}\left(\mathcal{H} \ominus \tilde{\mathcal{H}}_{k}\right) \\
& =\left(D_{\mathcal{N}_{k}} \otimes I_{\overline{\mathcal{N}}_{k}}\right)\left(\left(\mathcal{H}_{\mathcal{N}_{k}}^{t} \oplus \mathcal{H}_{\mathcal{N}_{k}}^{r}\right) \otimes \mathcal{H}_{\overline{\mathcal{N}}_{k}}\right) \\
& \subseteq\left(\mathcal{H}_{\mathcal{N}_{k}}^{t} \oplus \mathcal{H}_{\mathcal{N}_{k}}^{r}\right) \otimes \mathcal{H}_{\overline{\mathcal{N}}_{k}} .
\end{aligned}
$$

Furthermore,

$$
\bigcap_{k} \tilde{\mathcal{H}}_{k} \subset \tilde{\mathcal{H}}_{k}=\mathcal{H}_{\mathcal{N}_{k}}^{w} \otimes \mathcal{H}_{\overline{\mathcal{N}}_{k}} \perp\left(\mathcal{H}_{\mathcal{N}_{k}}^{t} \oplus \mathcal{H}_{\mathcal{N}_{k}}^{r}\right) \otimes \mathcal{H}_{\overline{\mathcal{N}}_{k}}
$$

Thus, by combining Eqs. (17) and (18), we obtain $D_{k} \mathcal{H}^{\prime} \perp \bigcap_{k} \tilde{\mathcal{H}}_{k}$, so by definition of $\mathcal{H}^{\prime}$ we have $D_{k} \mathcal{H}^{\prime} \subseteq$ $\mathcal{H}^{\prime}$.

Given Eq. (15) and the above proposition, we can then apply Theorem 3 to establish that the constructed dynamics makes $\rho_{d}$ conditional $\mathcal{H}^{\prime}$-DQLS. We summarize the results of this section in the following:

Corollary 6 Let $\rho_{d}$ and the given neighborhood structure $\left\{\mathcal{N}_{k}\right\}$ be such that Eq. (14) holds. Then by choosing $\mathcal{H}^{\prime}=\mathcal{H} \ominus \bigcap_{k}\left(\mathcal{H}_{\mathcal{N}_{k}}^{w} \otimes \mathcal{H}_{\overline{\mathcal{N}}_{k}}\right)$, and a set of $Q L\left\{D_{k}\right\}$ that satisfy Eq. (16), $\rho_{d}$ is $\mathcal{H}^{\prime}-D Q L S$.

As remarked, a notable example of states satisfying the property in Eq. (14) is the class of W states. Their stabilization using this technique will be explicitly addressed in Sec. VI. 


\section{Conditional stabilization under constraints on the initial attraction basin}

In this case, the additional assumption we impose on the noise operators $\left\{D_{k}\right\}$ is that both $\mathcal{H}^{\prime}$ and $\mathcal{H}^{\prime \perp}$ are left invariant. This enables us to test a candidate subspace $\mathcal{H}^{\prime}$ and construct associated QL $\left\{D_{k}\right\}$ by employing a variation of the randomized algorithm presented in Sec. IIIB for general QL stabilization.

Technically, the key simplification that the above invariance requirements translates into is the fact that only linear constraints are imposed on the noise operators. This may be seen by writing the block decomposition of the $D_{k}$ with respect to $\mathcal{H}=\mathcal{H}^{\prime} \oplus \mathcal{H}^{\prime \perp}$ :

$$
D_{k}=\left[\begin{array}{cc}
D_{S, k} & D_{P, k} \\
D_{Q, k} & D_{R, k}
\end{array}\right] .
$$

In order for $\mathcal{H}^{\prime}$ to be invariant, it must hold that [12]:

$$
\sum_{k} D_{S, k}^{\dagger} D_{P, k}=0
$$

and $D_{Q, k}=0$ for all $k$. In terms of the coefficients $\beta_{j k}$ that parametrize $D_{k}$ as in Eq. (11), the above Eq. (19) is clearly a set of quadratic equations. However, if $\mathcal{H}^{\prime \perp}$ is required to be invariant as well, then Eq. (19) becomes $D_{P, k}=0$ and all the constraints are linear. Hence, the proof of Theorem 2 (see Appendix VII) carries over to this case upon restricting to the subspace of $\hat{\beta}_{j k}$ such that $\rho_{d}, \mathcal{H}^{\prime}$, and $\mathcal{H}^{\prime \perp}$ are invariant. Let $\alpha_{j k}=0$ for all $j, k$. We thus have the following:

Theorem 4 If there exists a choice of $\beta_{j k}$ that makes $\rho_{d} \mathcal{H}^{\prime}-D Q L S$ and $\mathcal{H}^{\prime \perp}$ invariant, then almost any choice such that $\rho_{d}$ and $\mathcal{H}^{\prime \perp}$ are invariant makes $\rho_{d} \mathcal{H}^{\prime}-D Q L S$ as well.

We can thus provide the following randomized conditional stabilization algorithm:

Step 0: Finding a candidate $\mathcal{H}^{\prime}$. Given Lemmi 2 and 3 , we need $\mathcal{H}^{\prime} \supsetneq \mathcal{H}_{d}$ such that $\mathcal{H}^{\prime} \perp \mathcal{H}_{w}$. The largest, obvious candidate is of course $\mathcal{H}^{\prime}=\mathcal{H} \ominus \mathcal{H}_{w}$. A trial choice may otherwise be dictated by physical considerations and available experimental capabilities: e.g., in situations where a conserved quantity associated to an observable $\mathcal{O}$ exists, such that $\mathcal{H}_{d}$ and $\mathcal{H}_{w}$ belong to two different eigenvalues of $\mathcal{O}$, a way to construct $\mathcal{H}^{\prime}$ is to identify it with the eigenspace containing $\mathcal{H}_{d}$.

Step 1: Imposing quasi-locality of $\left\{D_{k}\right\}$. Pick, as before, a product operator basis for $\mathfrak{B}(\mathcal{H})$, say, $\left\{\sigma_{i_{1}} \otimes \ldots \otimes \sigma_{i_{n}} \mid i_{a}=1, \ldots, d_{a}^{2}\right\}$. Let $D_{k}$ be associated to a vector $\vec{d}_{k}$, whose components represent our free design parameters. Impose the QL requirement by demanding that the components associated to basis elements that are not QL vanish: let $\hat{B}_{k}$ be the orthogonal projection onto the subspace generated by the basis elements that are QL with respect to $\mathcal{N}_{k}$, and $\hat{B}_{k}^{\perp}=I-\hat{B}_{k}$. Write $\hat{D} \equiv\left[\vec{d}_{1}|\cdots| \vec{d}_{n}\right]$ for the matrix of all the free parameters in compact form. We must then require, for each $k$ :

$$
\hat{B}_{k}^{\perp} \vec{d}_{k}=0 .
$$

Step 2: Ensuring invariance of $\mathcal{H}_{0}$. Impose the linear constraints $D_{k}|\psi\rangle=0$ for all $k$. If $\hat{P}_{0}$ is the matrix representation of the linear (super)-operator $P_{0}(X)=X|\psi\rangle\langle\psi|$ with respect to the the chosen operator basis, this is equivalent to require, for each $k$ :

$$
\hat{P}_{0} \vec{d}_{k}=0 .
$$

Step 3: Ensuring invariance of $\mathcal{H}^{\prime}$ and $\mathcal{H}^{\prime \perp}$. We now impose a block-diagonal form for the $D_{k}$ with respect to the decompositon $\mathcal{H}=\mathcal{H}^{\prime} \oplus \mathcal{H}^{\prime \perp}$. That is, upon writing

$$
D_{k}=\left[\begin{array}{cc}
D_{S, k} & D_{P, k} \\
D_{Q, k} & D_{R, k}
\end{array}\right]
$$

we require that [12]:

$$
D_{Q, k}=0, \quad D_{P, k}=0,
$$

for all $k$. Note that Eq. (20) is a linear constraint, and can be imposed by requesting: $\left[D_{k}, \Pi^{\prime}\right]=0$, where $\Pi^{\prime}$ is the orthogonal projector associated to $\mathcal{H}^{\prime}$. In terms of the vectorization employed in the previous steps, this can in turn be rewritten as:

$$
\hat{P}^{\prime} \vec{d}_{k}=0,
$$

with $\hat{P}^{\prime}$ denoting the matrix representation of the super-operator associated with the commutator with $\Pi^{\prime}, \hat{P}^{\prime}(X)=\left[X, \Pi^{\prime}\right]$. For each $k$, the above (three) steps translate in the following (homogeneous) systems of linear equations:

$$
\hat{C} \vec{d}_{k}:=\left[\begin{array}{c}
\hat{B}_{k}^{\perp} \\
\hat{P}_{0} \\
\hat{P}^{\prime}
\end{array}\right] \vec{d}_{k}=0
$$

If a non-zero solution exists, then a subspace of solutions $\vec{d}_{k}$ exist, each corresponding to a QL operator $D_{k}$.

Step 4: Forcing convergence. Choose the free variables uniformly at random within a finite interval $[-\gamma, \gamma] \subset \mathbb{R}$.

We thus have the following Corollary as a direct consequence of Theorem 4 
Corollary 7 Let $\rho_{d}$ denote the target pure state. Assume that there exist an invariant subspace $\mathcal{H}^{\prime}$ and $Q L$ $\left\{D_{k}\right\}$ such that $\rho_{d}$ is $\mathcal{H}^{\prime}-D Q L S$ and $\mathcal{H}^{\prime \perp}$ is invariant. Then the above Steps 0-4 find a choice of $\mathcal{H}^{\prime}$ and $\left\{D_{k}\right\}$ with probability one.

From a practical standpoint, Proposition 7 provides an explicit algorithm to test whether a candidate subspace $\mathcal{H}^{\prime}$ makes the target $\rho_{d} \mathcal{H}^{\prime}$-DQLS and, if so, it also outputs a choice of effective noise operators $\left\{D_{k}\right\}$.

\section{QUASI-LOCAL STABILIZATION WITH DRIFT DYNAMICS}

In realistic scenarios, the uncontrolled system may evolve under an internal QL dynamics, due to a preexisting Hamiltonians and/or couplings with Markovian environments, resulting in a non-trivial, known drift generator, $\mathcal{L}_{0}\left(H_{0},\left\{L_{k}^{0}\right\}\right) \neq 0$, in the notation of Section $\amalg \mathrm{C}$ We first discuss the simpler case where only Hamiltonian drift is present $\left(L_{k}^{0} \equiv 0\right.$ for all $\left.k\right)$.

\section{A. Drift Hamiltonian}

We preliminarily note that if complete QL Hamiltonian control is available over $S$ with respect to the same locality notion of $H_{0}$ or a less restrictive one, then a control Hamiltonian $H_{c}=-H_{0}$ can be applied to undo the action of the drift, given that $H_{0}$ is known. In this case, the problem becomes again effectively drift-less, and the results developed in the previous sections apply. We thus assume here that limited (if any) Hamiltonian control is available.

In order to establish whether $\rho_{d}$ can be stabilized in the presence of $H_{0}$, we can first check whether $H_{0}|\Psi\rangle=\lambda|\Psi\rangle$, in which case the state is invariant. If not, the first step is to see whether invariance may be enforced by applying suitable QL dissipation. Consider a QL decomposition of $H_{0}=\sum_{k} H_{k}^{0}$, and decompose $H_{k}^{0}$ in matrix blocks according to $\mathcal{H}=\mathcal{H}_{d} \oplus \mathcal{H}_{d}^{\perp}$, that is:

$$
H_{k}^{0} \equiv\left[\begin{array}{ll}
H_{S, k} & H_{P, k} \\
H_{P, k}^{\dagger} & H_{R, k}
\end{array}\right]
$$

For each $k$, define:

$$
\tilde{D}_{k} \equiv\left[\begin{array}{cc}
1 & \tilde{D}_{P, k} \\
0 & 0
\end{array}\right], \quad \tilde{D}_{P, k}=2 i H_{P, k} .
$$

By Corollary 1 in [12], it follows that applying $\tilde{D}_{k}$ as a noise operator for each neighborhood makes $\rho_{d}$ invariant for the global dynamics. Therefore, by recalling Eqs. (5)(6) and Corollary 1, we can find an equivalent representation of the generator in standard form, say $\mathcal{L}\left(H_{0}^{\prime},\left\{D_{k}^{\prime}\right\}\right)$, with $H_{0}^{\prime}|\Psi\rangle=0$ and $\rho_{d}$ invariant under $\mathcal{L}$. We may then proceed as follows:
1. Determine whether $\rho_{d}$ would be DQLS in the absence of $H_{0}^{\prime}$, by applying Theorem 1 .

2. If $\rho_{d}$ is DQLS for $H_{0}^{\prime}=0$, a straightforward modification of Theorem 2 above (see also Corollary 8 in Appendix VII proves that a generic choice of stabilizing noise operators, $\left\{D_{k}\right\}$, will make $\rho_{d}$ GAS under the combined evolution generated by $\mathcal{L}\left(H_{0}^{\prime},\left\{D_{k}^{\prime}\right\} \bigcup\left\{D_{k}\right\}\right)$.

3. If $\rho_{d}$ is not DQLS for $H_{0}^{\prime}=0$ and some QL Hamiltonian control is available, we still invoke Theorem 2 to determine whether a stabilizing QL Hamiltonian $H_{c}$ (and possibly stabilizing noise operators $\left\{D_{k}\right\}$ ) can be found by randomizing the free parameters in the controlled generator. If $\rho_{d}$ is QLS and solutions are attainable with the available controls, with probability one a choice will be found, such that $\rho_{d}$ is GAS under the combined generator $\mathcal{L}\left(H_{0}^{\prime}+H_{c},\left\{D_{k}^{\prime}\right\} \bigcup\left\{D_{k}\right\}\right)$.

4. If $\rho_{d}$ is not DQLS and no Hamiltonian control is available, we need to check whether $H_{0}^{\prime}$ satisfies the necessary conditions of Proposition 3 (or Corollary 3). If so, we invoke Theorem 2 to determine whether stabilizing noise operators $\left\{D_{k}\right\}$ can be found by randomizing the free parameters in the controlled generator. If the drift Hamiltonian $H_{0}^{\prime}$ does not not obey Proposition 3 and $H_{c} \equiv 0, \rho_{d}$ cannot be made GAS.

5. If $\rho_{d}$ cannot be made GAS, conditional stabilization may be attempted, by invoking Theorem 4 and a suitable modification of the randomized stabilization algorithm. Specifically, in Step 0 it is necessary to additionally ensure that the candidate subspace $\mathcal{H}^{\prime}$ and its complement $\mathcal{H}^{\prime \perp}$ are invariant under the drift dynamics generated by $H_{0}^{\prime}$. By denoting with $\Pi^{\prime}$, as before, the orthogonal projector onto $\mathcal{H}^{\prime}$, this translates into requiring the additional compatibility requirement $\left[H_{0}^{\prime}, \Pi^{\prime}\right]=0$. If $\rho_{d}$ can be made $\mathcal{H}^{\prime}$-DQLS with $\mathcal{H}^{\prime}$ and $\mathcal{H}^{\prime \perp}$ invariant, the algorithm succeeds with probability one.

\section{B. Drift Hamiltonian and dissipation}

In the most general situation, the system may be driven by a QL Markovian drift dynamics, specified by a generator $\mathcal{L}_{0}\left(H_{0},\left\{L_{k}^{0}\right\}\right)$ with both $H_{0}$ and some noise generators being non-vanishing. In this case, the first key property to verify is whether the necessary condition for invariance of $\rho_{d}$ is obeyed by the dissipative drift, that is, whether for each neighborhood, we have $\mathcal{N}_{k}, L_{k}|\Psi\rangle=\lambda_{k}|\Psi\rangle$. If $L_{k}|\Psi\rangle \neq \lambda_{k}|\Psi\rangle$ for some $k$, then $\rho_{d}$ cannot be made GAS by the methods we described. While in practice one may expect that approximate stabilization be still meaningful and viable if the natural dissipation is sufficiently weak with respect to the available controlled dissipation, establishing rigorous results 
in this sense requires a separate analysis, which will be addressed elsewhere (see also [45] for relevant distance bounds).

Let us thus assume that $L_{k}|\Psi\rangle=\lambda_{k}|\Psi\rangle$ for all $k$. Then by using Corollary 1, we can write an equivalent generator in standard form, with operators $\left\{H_{0}^{\prime}, L_{k}^{\prime}\right\}$ such that $L_{k}^{\prime}|\Psi\rangle=0$. By the invariance requirement, these noise operators must satisfy Lemma 1 as well. In this way, we have effectively mapped the problem back the one just considered in Sec. VA, with only Hamiltonian drift. In case sufficient QL Hamiltonian control is available, we can remove the effect of $H_{0}^{\prime}$ as above, and the problem is reduced to determine whether $\rho_{d}$ is $D Q L S, Q L S$, or $\mathcal{H}^{\prime}$ $D Q L S$, as already discussed. Otherwise we may proceed as follows:

1. If the drift Hamiltonian $H_{0}^{\prime}$ cannot be canceled by Hamiltonian control, we need to determine whether it destabilizes the desired state. If so, the action of $H_{0}^{\prime}$ can be compensated in a way similar to the one given in Eq. (23), but, due to the non-zero dissipative drift $\left\{L_{k}^{\prime}\right\}$, we now need to choose

$\tilde{D}_{k} \equiv\left[\begin{array}{cc}1 & \tilde{D}_{P, k} \\ 0 & 0\end{array}\right], \quad \tilde{D}_{P, k}=2 i H_{P, k}-L_{S, k}^{\prime \dagger} L_{P, k}^{\prime}$.

The drift dynamics induced by $H_{0}^{\prime},\left\{L_{k}^{\prime}\right\}$, plus the QL operators $\left\{\tilde{D}_{k}\right\}$, can then be associated to a new generator $\mathcal{L}\left(H_{0}^{\prime \prime},\left\{L_{k}^{\prime \prime}\right\}\right) \equiv \mathcal{L}\left(H_{0}^{\prime \prime},\left\{L_{k}^{\prime}\right\} \bigcup\left\{\tilde{D}_{k}\right\}\right)$ in standard form, with $H_{0}^{\prime \prime}|\Psi\rangle=0$ and $\rho_{d}$ invariant under $\mathcal{L}$.

2. If $\rho_{d}$ is DQLS for $H_{0}^{\prime \prime}=0$, then by Corollary 8, a generic choice of stabilizing operators, $\left\{D_{k}\right\}$, will suffice to make $\rho_{d}$ GAS under the combined evolution generated by $\mathcal{L}\left(H_{0}^{\prime \prime},\left\{L_{k}^{\prime \prime}\right\} \bigcup\left\{D_{k}\right\}\right)$.

3. If $\rho_{d}$ is not DQLS for $H_{0}^{\prime \prime}=0$ and some QL Hamiltonian control is available, we can use Theorem 2 to determine whether a stabilizing QL Hamiltonian $H_{c}$ (and possibly stabilizing noise operators $\left.\left\{D_{k}\right\}\right)$ can be found by randomizing the free parameters in the controlled generator. If $\rho_{d}$ is QLS and solutions are attainable with the available controls, with probability one a choice will be found, such that $\rho_{d}$ is GAS under the combined generator $\mathcal{L}\left(H_{0}^{\prime \prime}+H_{c},\left\{L_{k}^{\prime \prime}\right\} \bigcup\left\{D_{k}\right\}\right)$.

4. If the state is not DQLS or $H_{0}^{\prime \prime}=0$ and no Hamiltonian control is available, we need to check whether $H_{0}^{\prime \prime}$ satisfies the necessary conditions of Proposition 3 (or Corollary 3). If so, we are left with the problem of finding stabilizing QL noise operators to be added to the internal ones, by randomizing the free parameters in the total controlled generator. If $H_{0}^{\prime \prime}$ has a destabilizing action on $\rho_{d}$, the state is not QLS.

5. If $\rho_{d}$ is neither DQLS nor QLS, conditional stabilization may be tested, in a way similar to what described for Hamiltonian drift only. In Step 0 of the stabilization algorithm, we now need to to ensure that both the candidate subspace $\mathcal{H}^{\prime}$ and $\mathcal{H}^{\prime \perp}$ are invariant under the full drift dynamics generated by $\mathcal{L}_{0}$. If $\rho_{d}$ can be made $\mathcal{H}^{\prime}$-DQLS with $\mathcal{H}^{\prime}$ and $\mathcal{H}^{\prime \perp}$ invariant, the algorithm succeeds with probability one.

\section{ILLUSTRATIVE EXAMPLES}

Consider a quantum register consisting of $n$ qubits, and let $\left\{\sigma_{i} \mid i=x, y, z\right\}$ denote single-qubit Pauli matrices [1], with the corresponding multi-qubit Pauli operators given by $\sigma_{i}^{(a)} \equiv I \otimes \ldots \otimes \sigma_{i} \otimes \ldots \otimes I \otimes I$, and $\sigma_{i}$ acting on the $a$-th qubit. Since the form of possible drift dynamics is highly system-dependent, we focus here on the drift-less setting, as relevant in particular to trapped-ion opensystem quantum simulators [4] and cold atomic gases [19].

\section{A. DQLS states}

As mentioned earlier in the text, the DQLS states include important classes of pure multipartite entangled states, most notably, all stabilizer and graph states, relative to the natural choice of neighborhoods associated with connected nodes on the graph [11, 36]. While characterizing the class of DQLS states for a pre-determined neighboorhood structure remains an interesting open problem, one can show that non-trivially entangled pure states which are provably not graph states are in DQLS. For instance, as already noted in [36], the DQLS set for $n=4$ qubits contains the symmetric Dicke state with two excitations,

$D_{4}^{(2)} \equiv(|0011\rangle+|0101\rangle+|1001\rangle+|0110\rangle+|1010\rangle+|1100\rangle) / \sqrt{6}$

which may be used as an optimal quantum resources in $1 \rightarrow 3$ telecloning protocols and has been experimentally characterized using polarization-entangled photons [39]. Imagine that the four qubits are arranged in a line. The state $D_{4}^{(2)}$ may be projected into both the three-qubit GHZ and the W class via single-qubit measurements. While the resulting $\mathrm{GHZ}_{3}$ and $\mathrm{W}_{3}$ states are graph states (hence DQLS) relative to the natural ('star'-connected) neighboorhood choice $\mathcal{N}_{1}=\{1,2\}$, $\mathcal{N}_{2}=\{2,1,3\}, \mathcal{N}_{3}=\{3,2\}$, this is no longer the case if a nearest-neighboorhood coupling constraint is imposed, in which case $\mathcal{N}_{1}=\{1,2\}, \mathcal{N}_{2}=\{2,3\}$.

In what follows, we explicitly address dissipative stabilization of $n$-qubit GHZ and W states, which play a paradigmatic role in QIP [55]. 


\section{B. Greenberger-Horne-Zeilinger cat states}

\section{GHZ states are not DQLS}

A representative of the $\mathrm{GHZ}$ class is the state $\rho_{\mathrm{GHZ}}=$ $|\Psi\rangle\langle\Psi|$, where

$$
\begin{aligned}
& |\Psi\rangle \equiv\left|\Psi_{\mathrm{GHZ}}\right\rangle \\
& =\left(|0\rangle^{\otimes n}+|1\rangle^{\otimes n}\right) / \sqrt{2} \equiv(|000 \ldots 0\rangle+|111 \ldots 1\rangle) / \sqrt{2} .
\end{aligned}
$$

As shown in [36], $\mathrm{GHZ}_{n}$ states are generally not DQLS, except in cases where the QL constraint becomes effectively trivial, as illustrated in the above three-qubit example. In fact, any reduced state on any (nontrivial) neighborhood is an equiprobable mixture of $|000 \ldots 0\rangle$ and $|111 \ldots 1\rangle$. It is then immediate to see that

$$
\mathcal{H}_{0}=\operatorname{span}\{|000 \ldots 0\rangle,|111 \ldots 1\rangle\}=\bigcap_{k} \operatorname{supp}\left(\rho_{\mathcal{N}_{\mathrm{k}}} \otimes \mathrm{I}_{\overline{\mathcal{N}}_{\mathrm{k}}}\right),
$$

with $\operatorname{dim}\left(\mathcal{H}_{0}\right)=d_{0}=2$. From the point of view of MPS theory, the GHZ state is known to be non-injective, and corresponding to the two-fold degenerate ground state of a frustration-free (gapped) Ising parent Hamiltonian [34].

\section{GHZ states can be QLS: conditions on the neighborhood} size

Let us explore under what conditions adding Hamiltonian control can render $\rho_{\mathrm{GHz}}$ GAS. Given Corollary 3 , we seek a QL Hamiltonian $H_{c}=\sum_{k} H_{k}$ such that:

$$
\begin{aligned}
& H_{c}\left|\Psi_{\mathrm{GZH}}\right\rangle=0, \\
& H_{c}\left|\Phi_{1}\right\rangle \notin \mathcal{H}_{0}, \quad\left|\Phi_{1}\right\rangle \equiv(|000 \ldots 0\rangle-|111 \ldots 1\rangle) / \sqrt{2} .
\end{aligned}
$$

In order for this to happen, $H_{c}$ must equivalently obey the condition

$$
H_{c}|000 \ldots 0\rangle=-H_{c}|111 \ldots 1\rangle \text {. }
$$

Since each component $H_{k}$ is QL, it acts non-trivially on at most a number $n_{k}$ of "symbols" (that is, 0 or 1 ) in the factorized states $|0\rangle^{\otimes n},|1\rangle^{\otimes n}$, respectively. Let $n$ be even and assume that $n_{k}<n / 2$. Then it follows that

$H_{c}|000 \ldots 0\rangle \in \operatorname{span}\left\{\left|x_{1}, \ldots, x_{n}\right\rangle, x_{j} \in\{0,1\}, \sum_{j} x_{j}<n / 2\right\}$,
$H_{c}|111 \ldots 1\rangle \in \operatorname{span}\left\{\left|x_{1}, \ldots, x_{n}\right\rangle, x_{j} \in\{0,1\}, \sum_{j} x_{j}>n / 2\right\}$.

Thus, the two vectors must be orthogonal, since they belong to subspaces spanned by two orthogonal sets of vectors. This means that a Hamiltonian satisfying the requirements of Corollary 3 does not exists. In other words, we need $H_{c}$ to be able to flip at least $n / 2$ qubits in the product basis states $|0\rangle^{\otimes n},|1\rangle^{\otimes n}$.
If we allow one neighborhood to include $n_{k}=n / 2$ qubits, we can always construct a QL Hamiltonian such that

$$
\begin{aligned}
& H_{c}(|000 \ldots 0\rangle=(|1 \ldots 10 \ldots 0\rangle-|0 \ldots 01 \ldots 1\rangle) / \sqrt{2} \\
& H_{c}(|111 \ldots 1\rangle=(-|1 \ldots 10 \ldots 0\rangle+|0 \ldots 01 \ldots 1\rangle) / \sqrt{2},
\end{aligned}
$$

with the vectors in the r.h.s. containing precisely $n / 2$ zeroes and $n / 2$ ones, which clearly satisfies the requirement. This may be achieved by considering a neighborhood structure consisting of two disjoint sets $\mathcal{S}_{\ell=1,2}$, each including half of the qubits if $n$ is even [or $(n+1) / 2,(n-$ $1) / 2$ in the odd- $n$ case], and QL Hamiltonian components given by

$$
H_{\ell}=(-1)^{\ell-1}\left(\prod_{a \in \mathcal{S}_{\ell}} \sigma_{x}^{(a)}\right) .
$$

If there exists two neighborhoods $\mathcal{N}_{k_{\ell}}$, compatible with the QL constraints, such that $\mathcal{S}_{\ell} \subset \mathcal{N}_{k_{\ell}}$, then, with a proper choice of the noise operators, a GHZ state may be rendered GAS. Otherwise, the above argument proves the following:

Proposition 5 Assume that no neighborhood exists, that contains $n / 2$ qubits if $n$ is even, or $(n+1) / 2$ if $n$ is odd. Then $\rho_{G H Z}$ is not $Q L S$.

While the randomized QLS algorithm may be applied if a large neighborhood exists, stabilizing generators may be constructed by inspection in simple low-dimensional situations. Let us reconsider, for example, $n=3$ qubits on a line, under the QL constraint of two-body nearestneighbor couplings. We may then choose the two disjoint sets $\mathcal{S}_{1}=\{1\}, \mathcal{S}_{2}=\{2,3\}$, and implement

$$
\begin{gathered}
H_{c}=\sigma_{x}^{(1)}-\sigma_{x}^{(2)} \otimes \sigma_{x}^{(3)}, \\
D_{1}=I \otimes(|00\rangle\langle 01|+| 11\rangle\langle 10|), \\
D_{2}=I \otimes(|00\rangle\langle 01|+i| 11\rangle\langle 10|) .
\end{gathered}
$$

We stress that the phase factor appearing in the Lindblad operator $D_{2}$ is not coincidental: in fact, the more symmetric choice $D_{2}^{\prime}=I \otimes(|00\rangle\langle 01|+| 11\rangle\langle 10|)$ would leave the -1-eigenspace of the operator $\bigotimes_{a=1,2,3} \sigma_{x}^{(a)}$ invariant for the Liouvillian generator associated to $H_{c}, D_{1}, D_{2}^{\prime}$. In constrast, $\rho_{\mathrm{GHZ}}$ is the unique invariant state for the generator associated to $H_{c}, D_{1}, D_{2}$, as required for GAS.

This simple example is sufficient to establish that DQLS $\subsetneq$ QLS, namely that there exist pure entangled states that are not stabilizable by dissipation alone but can indeed be made GAS by the addition of a suitable QL Hamiltonian. Furthermore, our argument for rendering GHZ states GAS with QL resources is general, and, in the light of Proposition 5] it requires neighborhoods of the minimum possible size. Since the latter scales linearly with the number of qubits $n$, the order of interaction in the required control Hamiltonians likewise grows extensively with $n$, preventing QLS to be achievable in a scalable fashion. 


\section{GHZ states are conditionally DQLS with a scalable strategy}

Consider again a three-qubit register, with locality notion associated to $\mathcal{N}_{1}=\{1,2\}$, and $\mathcal{N}_{2}=\{2,3\}$. In order to show that GHZ states can be $\mathcal{H}^{\prime}$-DQLS for a properly chosen $\mathcal{H}^{\prime}$, note that $|\Psi\rangle_{\mathrm{GHZ}}$ is a +1-eigenvector of $\sigma_{x}^{\otimes 3}$, whilst $\left|\Phi_{1}\right\rangle=(|000\rangle-|111\rangle) / \sqrt{2}$ is a -1-eigenvector of the same observable. In the notation of Section IV] we have $\mathcal{H}_{w}=\operatorname{span}\left\{\left|\Phi_{1}\right\rangle\right\}$. We can thus try to choose $\mathcal{H}^{\prime}$ as the +1 -eigenspace of $\sigma_{x}^{\otimes 3}$, denoted by $\mathcal{H}_{x x x}^{+}$. As noise operators, pick $D_{12}=D \otimes I$, and $D_{23}=I \otimes D$, with

$$
D=\left[\begin{array}{llll}
0 & 0 & 1 & 0 \\
0 & 0 & 0 & 0 \\
0 & 0 & 0 & 0 \\
0 & 1 & 0 & 0
\end{array}\right] \equiv \sigma_{+} \otimes \frac{\sigma_{z}+I}{2}-\sigma_{-} \otimes \frac{\sigma_{z}-I}{2},
$$

in the standard Pauli formalism. It is easy to show that they both commute with $\sigma_{x}^{\otimes 3}$, and leave $\rho_{\text {GHZ }}$ invariant. Finally, by running the DID algorithm for GAS verification described in Appendix VII starting from $\mathcal{H}_{0}=\operatorname{span}\{|000\rangle,|111\rangle\}$ as the target subspace, one finds that it runs to completion in 4 steps and hence $\mathcal{H}_{0}$ is GAS. Hence, by Theorem 3, we conclude that $\rho_{\mathrm{GHZ}}$ is

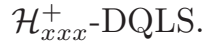

Does the same strategy still work for a generic number of qubits? More precisely, consider $n$ qubits arranged on a linear graph (equivalently, an open spin chain), with neighborhoods associated to all nearest-neighbor pairs, namely $\mathcal{N}_{k}=\{k, k+1\}_{k=1, \ldots, n-1}$. Implement a dissipator for each neighborhood of the form $D_{k}=D \otimes I_{\mathcal{N}_{k}}$, where $D$ has the structure given in (24). Consider, as in the above three-qubit case, any initial state with support on $\mathcal{H}^{\prime}$, the +1-eigenspace of the operator $\sigma_{x}^{\otimes n}$. Explicitly checking GAS of the $n$-qubit subspace $\mathcal{H}_{0}$ by resorting to the DID algorithm becomes impractical as $n$ grows. Luckily, Theorem 3 makes this step unnecessary. In fact, note that:

1. This choice of $D_{k}$ makes $\mathfrak{D}\left(\mathcal{H}_{\mathcal{N}_{k}}^{\circ}\right)$ GAS on any neighborhood, so that $\mathfrak{D}\left(\mathcal{H}_{0}\right)$ is GAS;

2. The $D_{k}$ commute with $\sigma_{x}^{\otimes n}$ and leave $\mathcal{H}^{\prime}$ invariant;

3. The other state in $\mathcal{H}_{0},\left|\Phi_{1}\right\rangle$ does not belong to $\mathcal{H}^{\prime}$.

We can thus directly conclude that the $\mathrm{GHZ}_{n}$ state is $\mathcal{H}^{\prime}$-DQLS, with the same choice of two-body dissipators for any $n$ and the dimension of the conditional preparation subspace being equal to $d / 2=2^{n-1}$. In practice, initialization to any state in $\mathcal{H}^{\prime}$ by either coherent or incoherent means will suffice. For instance, two possible scalable strategies are initialization into the product state $|+\rangle^{\otimes n}$ by application of a collective Hadamard gate $H^{\otimes n}$ to $|0\rangle^{\otimes n}$ (in case the latter is a natural starting point) or by a projective measurement of the collective (one-body) spin observable $S_{x} \equiv \sum_{a} \sigma_{x}^{(a)}$, post-selected on the outcome corresponding to the maximal eigenvalue (highest-weight) state.

\section{W states}

\section{W states are not $D Q L S$}

A representative of the $\mathrm{W}$ class is the state $\rho_{\mathrm{W}}=$ $|\Psi\rangle\langle\Psi|$, with

$|\Psi\rangle \equiv\left|\Psi_{\mathrm{W}}\right\rangle=(|100 \ldots 0\rangle+|010 \ldots 0\rangle+\ldots+|000 \ldots 1\rangle) / \sqrt{n}$.

This state is a permutation-invariant superposition of all computational basis states with a single 1, thus also a symmetric Dicke state one-excitation. The reduced states on any non-trivial neighborhood are statistical mixtures of $|000 \ldots 0\rangle$ and a smaller $\mathrm{W}$ state, say $\left|\Psi_{\mathrm{W}^{\prime}}\right\rangle$, whose dimension is determined by the neighborhood. Accordingly, as established in [36], $\rho_{\mathrm{W}}$ is generally not DQLS, since

$$
\mathcal{H}_{0}=\operatorname{span}\left\{|000 \ldots 0\rangle,\left|\Psi_{\mathrm{W}}\right\rangle\right\}=\bigcap_{k} \operatorname{supp}\left(\rho_{\mathcal{N}_{\mathrm{k}}} \otimes \mathrm{I}_{\overline{\mathcal{N}}_{\mathrm{k}}}\right)
$$

with $\operatorname{dim}\left(\mathcal{H}_{0}\right)=d_{0}=2$. Like the GHZ state, the $\mathrm{W}$ state is also known to be non-injective, however a frustrationfree gapped parent Hamiltonian does not exist in this case [34].

\section{W states can be QLS: two-body interactions}

We here show that $\mathrm{W}$ states can be made QLS in the presence of QL constraints that prevent DQLS to be achievable. Consider, in particular, a $n$-qubit register with neighborhoods associated to arbitrary subsystem pairs, that is, $\mathcal{N}_{j k}=\{j, k\}, j, k=1, \ldots, n, j \neq k$. Since $\mathcal{H}_{0}=\operatorname{span}\left\{\left|\Psi_{\mathrm{W}}\right\rangle,\left|0^{\otimes \mathrm{n}}\right\rangle\right\}$, we are in a situation where Corollary 3 applies.

In order to construct a control Hamiltonian that satisfies the requirements of Corollary B, it is useful to note that the target state may be rewritten as

$$
\begin{aligned}
\left|\Psi_{\mathrm{W}}\right\rangle= & \sqrt{\frac{n-2}{n}}|0\rangle\left|\Psi_{\mathrm{W}_{n-2}}\right\rangle|0\rangle \\
& +\frac{1}{\sqrt{n}}\left(|1\rangle|0\rangle^{\otimes(n-2)}|0\rangle\right. \\
& \left.+|0\rangle|0\rangle^{\otimes(n-2)}|1\rangle\right) .
\end{aligned}
$$

It is then easy to show that, for example, the following two-body Hamiltonian satisfies the desired conditions:

$$
H_{c}=\sigma_{x}^{(1)} P_{0}-P_{0} \sigma_{x}^{(n)}, \quad P_{0}=\sum_{a=2}^{n-1} \sigma_{z}^{(a)}-(n-4) I^{(a)} .
$$

This follows from the fact that $P_{0}$ is an operator on $\bigotimes_{a=2}^{n-1} \mathcal{H}_{a}$ which obeys

$$
P_{0}\left|\Psi_{W_{n-2}}\right\rangle=0, \quad P_{0}|0\rangle^{\otimes(n-2)}=|0\rangle^{\otimes(n-2)} .
$$


While we derived this particular Hamiltonian guided by simple symmetry considerations, to our scope it suffices to verify by direct computation that

$$
H_{c}\left|\Psi_{W}\right\rangle=0, \quad H_{c}|0\rangle^{\otimes n}=|10 \ldots 0\rangle-|0 \ldots 01\rangle,
$$

as needed for Corollary 3. In order to conclude that the above Hamiltonian indeed makes $\left|\Psi_{W}\right\rangle$ QLS, we also need to exhibit an explicit choice of dissipators $D_{k}$. To this aim, consider in each two-qubit neighborhood a ladder dissipator of the form

$$
D=\left[\begin{array}{llll}
0 & 1 & 1 & 0 \\
0 & 0 & 0 & 1 \\
0 & 0 & 0 & 1 \\
0 & 0 & 0 & 0
\end{array}\right] \equiv I \otimes \sigma_{+}+\sigma_{+} \otimes I
$$

This choice makes the set of states with support on $\mathcal{H}_{\mathcal{N}_{k}}^{\circ}$ GAS on each neighborhood, and hence $\mathcal{H}_{0}$ is stabilized.

Explicit numerical calculation demonstrates that the above combination of Hamiltonian and dissipative control makes $\left|\Psi_{W}\right\rangle$ GAS, and hence QLS, at least for $n=3,4$. While we have no general formal proof, we expect that the same protocol will work for arbitrary $n$. This shows how W states can be made QLS by allowing for arbitrary two-body interactions. Whether scalable stabilization protocols may be constructed in other relevant QL scenarios, for instance solely involving nearestneighbors two-body interactions, remains open to further investigation.

\section{W states are conditionally DQLS with a scalable strategy}

Interestingly, W states can be conditionally stabilized by employing the systematic approach of Section IVB.

The key step is to show that the strict inclusion condition given in Eq. (14) holds. As we already noticed, with respect to any neighboorhood topology we have $\mathcal{H}_{0}=\operatorname{span}\left\{\left|\Psi_{W}\right\rangle,|0\rangle^{\otimes n}\right.$. $\}$ Let $d_{k}$ denote the number of subsystems in the neighboorhood $\mathcal{N}_{k}$ and, as before, let $\left|\Psi_{W_{d_{k}}}\right\rangle$ be a W state on $d_{k}$ qubits. We thus have to check, if for every $k$ :

$$
\begin{aligned}
\operatorname{supp}\left(\operatorname{Tr}_{\overline{\mathcal{N}}_{\mathrm{k}}}\left(|0\rangle\left\langle\left. 0\right|^{\otimes \mathrm{n}}\right)\right)\right. & =\operatorname{span}\left\{|0\rangle^{\otimes d_{k}}\right\} \\
& =\mathcal{H}_{\mathcal{N}_{k}}^{w} \subsetneq \mathcal{H}_{\mathcal{N}_{k}}^{\circ} \\
& =\operatorname{span}\left\{\left|\Psi_{W_{d_{k}}}\right\rangle,|0\rangle^{\otimes d_{k}}\right\},
\end{aligned}
$$

which is clearly true. Consider then

$$
\begin{aligned}
\mathcal{H}^{\prime}=\mathcal{H} \ominus \bigcap_{k} \tilde{\mathcal{H}}_{k} & =\mathcal{H} \ominus \bigcap_{k} \operatorname{span}\left\{|0\rangle^{\otimes d_{k}}\right\} \otimes \mathcal{H}_{\overline{\mathcal{N}}_{k}} \\
& =\mathcal{H} \ominus \operatorname{span}\left\{|0\rangle^{\otimes n}\right\} .
\end{aligned}
$$

Physically, we may think of $\mathcal{H}^{\prime}$ as the subspace of states orthogonal to the vacuum, thus initialization in $\mathcal{H}^{\prime}$ may be achieved in principle by any (coherent or incoherent) means that creates at least one "excitation".
In each neighborhood, consider now $|0\rangle^{\otimes d_{k}},\left|\Psi_{W}^{d_{k}}\right\rangle$ in this order and complete it to an orthonormal basis for $\mathcal{H}_{\mathcal{N}_{k}}$. With respect to this basis, define the following ladder-type Lindblad operator:

$$
D_{\mathcal{N}_{k}}=\left[\begin{array}{c|c|ccc}
0 & 0 & & \cdots & \\
\hline 0 & 0 & 1 & 0 & \cdots \\
\hline \vdots & 0 & 0 & 1 & \ddots \\
& & & \ddots & \ddots
\end{array}\right] .
$$

The corresponding dissipative process may be thought as cooling the system to the reduced W state $\left|\Psi_{W}^{d_{k}}\right\rangle$, while leaving the (QL) ground state $|0\rangle^{\otimes d_{k}}$ invariant. Then construct the overall dissipators as $D_{k}=D_{\mathcal{N}_{k}} \otimes I_{\overline{\mathcal{N}}_{k}}$. This choice ensures that:

1. $\mathcal{H}^{\prime}$ is invariant for each $D_{k}$;

2. $\mathfrak{D}\left(\mathcal{H}_{0}\right)$ is GAS.

Corollary 6 may then be invoked to establish that $\left|\Psi_{W}\right\rangle$ is $\mathcal{H}^{\prime}$-DQLS for the choice of $\mathcal{H}^{\prime}$ and $\left\{D_{k}\right\}$ we made. Remarkably, the proposed protocol is both scalable and portable, in the sense it works for an arbitrary number of qubits and arbitrary QL notions.

\section{CONCLUSION AND OUTLOOK}

We have provided a system-theoretic analysis of different scenarios and strategies for designing Markovian evolutions that have a desired pure entangled state as their unique stable steady state, using time-independent control parameters and subject to realistic locality constraints. In particular, we have shown how target states that are not stabilizable under purely dissipative QL control, as previously considered in [36], may be dissipatively prepared upon restricting the allowed set of initializations or by allowing a combination of Hamiltonian and dissipative control. We have further addressed the role of Hamiltonian and/or dissipative drift dynamics, as arising from possible always-on coherent interactions and/or couplings of the target system to an uncontrollable Markovian environment. Constructive algorithms for synthesizing effective choices of stabilizing Markovian generators have been presented, suitable in principle for open-loop control implementations based on a switching output-feedback law along the lines described in [36]. In particular, if the Markovian semigroup is obtained as an average over the trajectories of a stochastic master equation, we recall that convergence of the semigroup entails convergence of the underlying stochastic dynamics in probability [4].

While our results substantially expand the theoretical framework and toolbox for QL dissipative entanglement engineering in QIP, a number of open questions and further directions for exploration exist. For timeindependent Markovian dynamics as considered thus far, 
issues of efficiency and robustness are especially important from a practical standpoint: (i) On the one hand, once a target state of interest is found to be stabilizable, it is desirable to characterize the speed with which asymptotic convergence is attained depending on the system size and the elapsed (in reality always finite) stabilization time. While the analysis carried out in [14] indicates that the relevant Liouvillian spectral gap scales favorably with $n$ at least for injective MPSs, additional work is needed for more general classes of states as well as for optimally "tuning" the convergence speed as a function or the available control parameters, in the spirit of [35]. (ii) On the other hand, assessing how sensitive steady states are with respect to deviations of the actual control parameters with respect to the intended ones, and/or to additional (static or time-dependent, possibly quantitatively unspecified) perturbations is worth being pursued both in terms of analytical bounds and numerical exploration in specific QIP settings. General results recently established in [45, 46] may prove useful in that respect.

Partly related to the above, given that finite evolution time and errors will typically prevent a pure steady state to be exactly achieved, addressing the general problem of dissipative QL mixed-state stabilization is also a natural important next step. Additional physical motivation to consider target mixed entangled states is provided by the possibility to characterize and engineer non-equilibrium critical behavior in noise-driven many-body systems, so- called dissipative phase transitions, as recently investigated e.g. in [40 43] and already experimentally explored in [5] in small dimension.

Finally, a yet different direction is provided by the investigation of dissipative entanglement engineering in different classes of open quantum dynamical models: from this point of view, both switched QL Lindblad dynamics generalizing on the work of [6], and discrete-time Kraus-map engineering [47] are especially promising in the near term. Developing a stabilization framework for continuous-time non-Markovian dynamics is also an important challenge down the line, as relevant to both understanding the role of non-Markovianity as a resource [48] and to open-system quantum simulators [4, 49, 50].

\section{Acknowledgements}

L.V. is grateful to Gerardo Ortiz for discussions and Norbert Schuch for input on MPS theory. F.T. and L.V. acknowledge support by the QUINTET and the QFuture projects of the University of Padova, Italy. L.V. acknowledges partial support from the NSF through grant number PHY-1104403 and hospitality and partial support from the Kavli Institute of Theoretical Physics at UCSB, where part of this work was completed.
[1] M. A. Nielsen and I. L. Chuang (2000), Quantum Computation and Quantum Information. Cambridge University Press.

[2] J. P. Dowling and G. Milburn (2003), Phil. Trans. R. Soc. London A 361, 1655.

[3] S. Lloyd and L. Viola (2001), Phys. Rev. A 65, 010101 (R).

[4] J. T. Barreiro, M. Müller, P. Schindler, D. Nigg, T. Monz, M. Chwalla, M. Hennrich, C. F. Roos, P. Zoller, and R. Blatt (2011), Nature 470, 486.

[5] P. Schindler, M. Müller, D. Nigg, J. T. Barreiro, E. A. Martinez, M. Henrich, T. Monz, S. Diehl, P. Zoller, and R. Blatt (2012), arXiv:1212.2418.

[6] V. Bergholm and T. Schulte-Herbrüggen (2012), arXiv:1206.4945.

[7] A. R. R. Carvalho, P. Milman, R.L. de Matos Filho, and L. Davidovich (2001), Phys. Rev. Lett. 86, 4988.

[8] J. F. Poyatos, J.I. Cirac and P. Zoller (1996), Phys. Rev. Lett. 77, 4728.

[9] A. N. Boto , P. Kok, D. S. Abrams, S. L. Braunstein, C. P. Williams, and J. P. Dowling (2000), Phys. Rev. Lett. 85, 2733.

[10] S. Diehl, A. Micheli, A. Kantian, B. Kraus, H. P. Büchler, and P. Zoller (2008), Nature Phys. 4, 878.

[11] B. Kraus, S. Diehl, A. Micheli, A. Kantian, H. P. Büchler, and P. Zoller (2008), Phys. Rev. A 78, 042307.

[12] F. Ticozzi and L. Viola (2008), IEEE Trans. Aut. Contr. 53, 2048.

[13] F. Ticozzi and L. Viola (2009), Automatica 45, 2002.
[14] F. Verstraete, M. M. Wolf, and J. I. Cirac (2009), Nature Phys. 5, 633.

[15] S. G. Schirmer and X. Wang (2010), Phys. Rev. A 81, 062306.

[16] F. Ticozzi, S. G. Schirmer, and X. Wang (2010), IEEE Trans. Aut. Contr. 55, 2901.

[17] J. Busch, S. De, S. S. Ivanov, B. T. Torosov, T. P. Spiller, and A. Beige (2011), Phys. Rev. A 84, 022316.

[18] A. Pechen (2011), Phys. Rev. A 84, 042106.

[19] S. Diehl, E. Rico, M. A. Baranov, and P. Zoller (2011), Nature Phys. 7, 971.

[20] X.-Yu Chen, L.-T. Shen, Z.-B. Yang, H.-Z. Wu, and M.F. Chen (2012), J. Opt. Soc. Am. B 29, 1535.

[21] M. Kiffner, U. Dorner, and D. Jaksch (2012), Phys. Rev. A 85, 023812.

[22] E. G. Dalla Torre, J. Otterbach, E. Demler, V. Vuletic, and M. D. Lukin (2012), arXiv:1209.1991.

[23] N. Yamamoto (2012) Phil. Trans. R. Soc. London A 370, 5324.

[24] Y. Ikeda and N. Yamamoto (2012), arXiv:1211.5788.

[25] Y.-D. Wang and A. A. Clerk (2013), arXiv:1301.5553.

[26] H. Krauter, C.A Muschik, W. Wasilewski, J.M. Petersen, J.I. Cirac and E.S. Polzik (2011), Phys. Rev. Lett. 107, 080503.

[27] C. A. Muschik, E. S. Polzik, and J. I. Cirac (2011), Phys. Rev. A 83, 052312.

[28] Z. Leghtas, U. Vool, S. Shankar, M. Hatridge, S.M. Girvin, M.H. Devoret, M. Mirrahimi (2013), arXiv:1303.3819 
[29] R. Alicki and K. Lendi (1987), Quantum dynamical semigroups and applications, Springer (Berlin).

[30] M. Fannes, B. Nachtergale, and R. F. Werner (1992), Commun. Math. Phys. 144, 443.

[31] J. P. LaSalle, (1987). The Stability of Dynamical Systems, SIAM Regional Conference Series in Applied Mathematics, Hamilton Press, New Jersey.

[32] D. Perez-Garcia, F. Verstraete, M. M. Wolf, and J. I. Cirac (2007), Quantum Inf. Comput. 7, 401.

[33] D. Perez-Garcia, F. Verstraete, M. M. Wolf, and J. I. Cirac (2008), Quant. Inf. Comp. 8, 0650.

[34] C. Fernandez-Gonzales, N. Schuch, M. M. Wolf, J. I. Cirac, and D. Perez-Garcia (2011), arXiv:1210.6613.

[35] F. Ticozzi, R. Lucchese, P. Cappellaro, and L. Viola (2011), IEEE Trans. Autom. Contr. 53, 2048.

[36] F. Ticozzi and L. Viola (2012), Phil. Trans. R. Soc. London A 370, 5259.

[37] Preliminary results were presented in: F. Ticozzi and L. Viola (2012), Proceedings of the International Federation of Automatic Control, Lagrangian and Hamiltonian Methods for Non-Linear Control, Vol. 4, 220.

[38] R. A. Horn and C. R. Johnson (1990), Matrix Analysis, Cambridge University Press (New York).

[39] N. Kiesel, C. Schmid, G. Tóth, E. Solano, and H. Weinfurter (2007), Phys. Rev. Lett. 98, 063604.

[40] H. J. Carmichael (1980), J. Phys. B 13, 3551.

[41] J. Eisert and T. Prosen (2012), arXiv:1012.5013

[42] B. Horstmann, J. I. Cirac, and G. Giedke (2013), Phys. Rev. A 87, 012108.

[43] H. Schwager, J. I. Cirac, and G. Giedke (2013), Phys. Rev. A 87, 022110.

[44] F. Ticozzi, K. Nishio, C. Altafini (2013), IEEE Trans. Aut. Contr. 58, 74.

[45] O. Szehr and M. Wolf (2012), arXiv:1210.1171.

[46] P. Rouchon and A. Sarlette (2013), arXiv:1302.6899.

[47] G. Baggio, F. Ticozzi, and L. Viola (2012), Proceedings of the 51st Control and Decision Conference, pp. 10721077.

[48] S. F. Huelga, A. Rivas, and M. B. Plenio (2012), Phys. Rev. Lett. 108, 160402.

[49] S. Mostame, P. Rebentrost, A. Eisfeld, A. J. Kerman, D. I. Tsomokos, and A. Aspuru-Guzik (2012), New J. Phys. 14, 105013.

[50] A. Chiuri, C. Greganti, L. Mazzola, M. Paternostro, and P. Mataloni (2012), Sci. Reports 2, 968.

[51] This follows directly from the fact that Eq. (1) defines a linear autonomous dynamical system, as long as the generator $\mathcal{L}$ is time-independent as assumed. For more general dynamical systems, different notions of stability may be relevant, see e.g. Ref. [31].

[52] Note that in this simple setting, if the target state admits a partial factorization (e.g., a product of two entangled states), there is no loss of generality in stabilizing such factors individually, possibly at the expenses of redefining the neighborhood structure to make it compatible with the given factorization, as noted in [36].

[53] This class includes all so-called (blocked) injective PEPSs or MPSs. A non-injective MPSs is the degenerate ground state of a frustration-free parent Hamiltonian if all the matrices in the MPS description can be blockdiagonalized to a form where each block corresponds to an injective MPS. However, there exists non-injective MPSs that are still the unique ground state of their parent Hamiltonian.
[54] Since we consider real linear combinations, the dimension is doubled with respect to the more usual complex field, see Appendix B.

[55] We remark that dissipative preparation of entangled states sufficiently close to GHZ and/or W states (practical stabilization in the control-theoretic jargon), has been analyzed in specific physical scenarios, see e.g. [17, 20].

: Linear-algebraic results

We establish here a preliminary result that will be instrumental in the proof of the main Theorem 2, Let us first recall a basic result from the theory of analytic functions:

Lemma 4 Let $f(x): \mathbb{R}^{K} \rightarrow \mathbb{R}^{M}$ be a (non-zero) analytic function in $x \in \mathbb{R}^{K}$ and let $\mathcal{S}=\left\{x \in \mathbb{R}^{K} \mid f(x)=0\right\}$. Then $\mu(\mathcal{S})=0$, where $\mu$ is the Lebesgue measure in $\mathbb{R}^{K}$.

Define an $m \times n$ matrix $X=\left[f_{j k}(x)\right]$, with $f_{j k}: \mathbb{R}^{K} \rightarrow$ $\mathbb{C}$, such that the real and imaginary parts $\Re\left(f_{j k}\right), \Im\left(f_{j k}\right)$ are (real)-analytic, and let $r_{m} \equiv \max _{x \in \mathbb{C}^{K}} \operatorname{rank}(X)$. Notice that $\operatorname{rank}(X) \in\{0, \ldots, \min \{n, m\}\}$ for all $x \in \mathbb{C}^{K}$, and the maximum is attained in $\mathbb{R}^{K}$. We thus have the following:

Lemma 5 The set $\mathcal{X}=\left\{x \in \mathbb{R}^{K} \mid \operatorname{rank}(\mathrm{X})<\mathrm{r}_{\mathrm{m}}\right\}$ is such that $\mu(\mathcal{X})=0$.

Proof: Let $\hat{x} \in \mathbb{R}^{K}$ be such that $\hat{X}=\left[f_{j k}(\hat{x})\right]$ has $\operatorname{rank}(\hat{X})=r_{m}$. Then there exists a square $r_{m} \times r_{m}$ submatrix $X_{m}$ of $\hat{X}$ that has rank $r_{m}$ [38]. Notice that $\operatorname{det}\left(X_{m}\right)$ is a (complex) polynomial function of the elements of $X, f_{j k}$, and hence of $\Re\left(f_{j k}\right), \Im\left(f_{j k}\right)$. Thus, $\Re\left(\operatorname{det}\left(X_{m}\right)\right)$ and $\Im\left(\operatorname{det}\left(X_{m}\right)\right)$ are (real)-analytic functions of $x \in \mathbb{R}^{n}$, and by virtue of the previous Lemma the set:

$$
\begin{aligned}
\mathcal{X} & =\left\{x \in \mathbb{R}^{K} \mid \operatorname{det}\left(X_{m}\right)=0\right\} \equiv\left\{x \in \mathbb{R}^{K} \mid \Re\left(\operatorname{det}\left(X_{m}\right)\right)\right. \\
& \left.=0=\Im\left(\operatorname{det}\left(X_{m}\right)\right)\right\} \\
& =\left\{x \in \mathbb{R}^{K} \mid \operatorname{rank}(X)<r_{m}\right\}
\end{aligned}
$$

is the intersection of two zero-measure set. Thus, it itself satisfies $\mu(\mathcal{X})=0$.

: Dissipation-induced decomposition and randomized stabilization

A general approach to decide the stability of a subspace, or more precisely, of the set of states with support on a subspace, has been developed in [35]. We recall here some basic ideas and results, expanding the (partially randomized) strategy for Hamiltonian control design proposed there to the synthesis of both coherent and dissipative controls.

Let $\mathcal{H}_{S}$ be a proper subspace of $\mathcal{H}$ and $\mathfrak{D}\left(\mathcal{H}_{S}\right) \subsetneq \mathfrak{D}(\mathcal{H})$ the set of states with support on $\mathcal{H}_{S}$. Then $\mathfrak{D}\left(\mathcal{H}_{S}\right)$ is GAS for the QDS dynamics if and only if a Hilbert space decomposition in orthogonal subspaces, of the form

$$
\mathcal{H}=\mathcal{H}_{S} \oplus \mathcal{H}_{T}^{(1)} \oplus \mathcal{H}_{T}^{(2)} \ldots \oplus \mathcal{H}_{T}^{(q)},
$$


can be obtained as the output of a constructive algorithm for GAS verification 35] (see also next section). Such decomposition is called the Dissipation-Induced Decomposition (DID). Each of the subspaces $\mathcal{H}_{T}^{(i)}$ in the direct sum is referred to as a basin.

Partitioning each matrix associated to the noise operators $D_{k}$ in blocks according to the DID results in the following standard structure, where the upper blockdiagonal blocks establish the dissipation-induced, cascade connections between the different basins $\mathcal{H}_{T}^{(i)}$ :

$$
D_{k}=\left[\begin{array}{c|cccc}
D_{S} & \hat{D}_{P}^{(0)} & 0 & \ldots & \\
\hline 0 & D_{T}^{(1)} & \hat{D}_{P}^{(1)} & 0 & \cdots \\
\vdots & D_{Q}^{(1)} & D_{T}^{(2)} & \hat{D}_{P}^{(2)} & \ddots \\
& \vdots & \ddots & \ddots & \ddots
\end{array}\right]_{k} .
$$

Similarly, for the control Hamiltonian we get:

$$
H_{c}=\left[\begin{array}{c|ccc}
H_{S} & H_{P}^{(0)} & 0 & \cdots \\
\hline H_{P}^{(0) \dagger} & H_{T}^{(1)} & \cdots & \\
0 & \vdots & \ddots & \\
\vdots & & &
\end{array}\right]_{k} .
$$

By construction, the $\hat{D}_{P}^{(i)}$ blocks are either zero or full rank. The fact that the first column of blocks has only $D_{S} \neq 0$ is a necessary condition for the invariance of $\mathfrak{D}\left(\mathcal{H}_{S}\right)$. It follows that $\hat{D}_{P}^{(0)} \neq 0$, otherwise $\mathfrak{D}\left(\mathcal{H}_{S}\right)$ cannot be GAS.

Consider now the target pure state $\rho_{d}=|\Psi\rangle\langle\Psi|$, its support $\mathcal{H}_{d} \equiv \mathcal{H}_{S}, \mathcal{H}_{d}^{\perp} \equiv \mathcal{H}_{R}$, and $\mathcal{H}=\mathcal{H}_{S} \oplus \mathcal{H}_{R}$. Assume that we are free to design $H,\left\{D_{k}\right\}$ in parametric form, as given in Eq. (11). The DID provides a key tool for proving Theorem 2 which we reproduce below:

Theorem 2 If there exists a choice of $\alpha_{j k}, \beta_{j k} \in \mathcal{I}=$ $[-\gamma, \gamma]$ that makes $\rho_{d} Q L S$, then almost any choice of $\alpha_{j k}, \beta_{j k} \in \mathcal{I}$ that makes $\rho_{d}$ invariant, makes it $Q L S$ as well.

Proof: We begin by observing that if a choice of controls $H_{c},\left\{D_{k}\right\}$ make $\mathfrak{D}\left(\mathcal{H}_{S}\right)$ GAS, then for any $\lambda \in \mathbb{R}$ the rescaled controls $\lambda H_{c},\left\{\sqrt{\lambda} D_{k}\right\}$ also make it GAS, since the total Liouvillian generator is linear in $H_{c}$ and quadratic in $D_{k}$. Assume then that a certain choice of $\alpha_{j k}, \beta_{j k}$ makes $\mathfrak{D}\left(\mathcal{H}_{S}\right)$ invariant: one can always rescale all $\alpha_{j k}, \beta_{j k}$ by $\lambda$ small enough so that they are all in $[-\gamma, \gamma]$ and still obtain a viable solution. Hence, it is not restrictive to look for stabilizing parameters in a bounded interval.

Next, assume that $\alpha_{j k}, \beta_{j k}$ make $\mathfrak{D}\left(\mathcal{H}_{S}\right)$ invariant. Since, as shown in Section IIB it is not restrictive to assume that $H_{c},\left\{D_{k}\right\}$ are in standard form, we may take $\mathcal{H}_{S} \subseteq \operatorname{ker}\left(D_{k}\right)$ for all $k$. The invariance conditions then translate in linear constraints on $\alpha_{j k}, \beta_{j k}$, namely:

$$
\sum_{j} \alpha_{j k} \sigma_{j k}|\Psi\rangle=0, \quad \sum_{j} \beta_{j k} \sigma_{j k}|\Psi\rangle=0,
$$

Since by hypothesis $\rho_{d}$ is GAS, there must exist at least a solution. If the solution is unique, the statement is trivial. If the solution is not unique, by linearity there is a subspace of solutions. If so, we may re-parametrize the admissible solutions in the free-parameters $\hat{\alpha}_{j k}, \hat{\beta}_{j k} \in \mathbb{R}$.

We now focus on a DID that makes $\rho_{d}$ GAS, and that will be proved to be generic. By construction, the matrix block decomposition of the matrix representation of $H_{c},\left\{D_{k}\right\}$ must be such that for each iteration, indexed by $j$, either

$$
\tilde{\mathcal{D}}^{(j)}:=\left[\begin{array}{c}
D_{P, 1}^{(j)} \\
\vdots \\
D_{P, M}^{(j)}
\end{array}\right]
$$

has maximum rank $r^{(j)}=\max \left\{\operatorname{dim}\left(\mathcal{H}_{T}^{(j-1)}\right), \operatorname{dim}\left(\mathcal{H}_{T}^{(j)}\right)\right\}$ (see Step 2, 3.a and 3.b), or

$$
\tilde{\mathcal{L}}_{P}^{(j)}:=i H_{P}^{(j)}-\frac{1}{2} \sum_{k} D_{Q, k}^{(j) \dagger} D_{T, k}^{(j)}
$$

has full rank $r^{(j)}$ (defined as above, see step 3.c of the algorithm). At the $j$-th iteration, $\operatorname{dim}\left(\mathcal{H}_{T}^{(j-1)}\right)$ is fixed, but we can choose a set of parameters $\hat{\alpha}_{j k}, \hat{\beta}_{j k}$ that maximizes $\operatorname{dim}\left(\mathcal{H}_{T}^{(j)}\right)$. Given Eq. (29)-(30), this is equivalent to maximize the rank of either $\tilde{\mathcal{D}}^{(j)}$ or $\tilde{\mathcal{L}}_{P}^{(j)}$ at each iteration. The elements of $\tilde{\mathcal{D}}^{(j)}$ and $\tilde{\mathcal{L}}_{P}^{(j)}$ are (complex) polynomial functions of the real parameters $\hat{\alpha}_{j k}, \hat{\beta}_{j k}$, and hence Lemma 5 ensures that the parameter set corresponding to maximal rank of the corresponding matrices has measure 1. This implies, in turn, that the same DID is constructed with probability one when the parameters are randomly chosen as above, establishing that the target can be made GAS with probability one, as claimed.

Along similar lines, we may show that the set of parameters that makes $\rho_{d}$ invariant has measure zero. In fact, such parameter choices correspond to the proper linear hyperplane determined by Eq. (28). The same holds for the DQLS property: the proof is identical to the one above, just consider the $\alpha_{j k}$ fixed. Thus, if there exists a choice of $\beta_{j k}$ that makes $\rho_{d}$ DQLS, then almost any choice of $\beta_{j k}$, such that $\rho_{d}$ is invariant, makes it DQLS as well.

Corollary 8 Assume $H_{c}=\sum_{k} H_{k}$ to be fixed, that is, $\alpha_{j k}$ are given for all $j, k$. If there exists a choice of $\beta_{j k} \in I$ that makes $\rho_{d} D Q L S$, then almost any choice of $\beta_{j k} \in I$, such that $\rho_{d}$ is invariant, makes it DQLS as well.

As discussed in the main text, the randomized approach carries over to conditional stabilization also, provided that care is taken in ensuring that no quadratic constraints appear. While imposing invariance of both $\mathcal{H}^{\prime}$ and its complement $\mathcal{H}^{\prime \perp}$ is a mathematically natural 
restriction to circumvent this issue, it need not always be easy to ensure in physical systems, especially in the presence of drift dynamics. Thus, an interesting open question is to determine whether a randomized approach may be devised for conditional DQLS in full generality.

: DID algorithm

In order to make our presentation self-contained, we reproduce here the algorithm for the construction of the DID 35]. The initial (target) subspace $\mathcal{H}_{d} \equiv \mathcal{H}_{S}$ (or, better, $\mathfrak{D}\left(\mathcal{H}_{S}\right)$ ), is GAS if and only if the algorithm runs to completion.

The inputs are a QDS generator $\mathcal{L}$ specified by the operators $H,\left\{L_{k}\right\}$ and an invariant subspace $\mathcal{H}_{S}$ or the evolution, that is, such that $e^{\mathcal{L} t} \mathfrak{D}\left(\mathcal{H}_{S}\right) \subseteq \mathfrak{D}\left(\mathcal{H}_{S}\right)$. Checking whether the above property holds can be easily done using Proposition 1 .

Algorithm for GAS verification and DID construction

Let $\mathcal{H}_{S}$ be invariant. Call $\mathcal{H}_{R}^{(0)}:=\mathcal{H}_{R}, \mathcal{H}_{S}^{(0)}:=\mathcal{H}_{S}$, choose an orthonormal basis for the subspaces and write the matrices with respect to that basis. Rename the matrix blocks as follows: $H_{S}^{(0)}:=H_{S}, H_{P}^{(0)}:=H_{P}, H_{R}^{(0)}:=$ $H_{R}, L_{S, k}^{(0)}:=L_{S, k}, L_{P, k}^{(0)}:=L_{P, k}$, and $L_{R, k}^{(0)}:=L_{R, k}$.

For $j \geq 0$, consider the following iterative procedure:

1. Compute the matrix blocks $L_{P, k}^{(j)}$ according to the decomposition $\mathcal{H}^{(j)}=\mathcal{H}_{S}^{(j)} \oplus \mathcal{H}_{R}^{(j)}$.

2. Define $\mathcal{H}_{R}^{(j+1)}:=\bigcap_{k} \operatorname{kerL}_{\mathrm{P}, \mathrm{k}}^{(\mathrm{j})}$.

3. Consider the following three sub-cases:

a. If $\mathcal{H}_{R}^{(j+1)}=\{0\}$, define $\mathcal{H}_{T}^{(j+1)}:=\mathcal{H}_{R}^{(j)}$. The iterative procedure is successfully completed. b. If $\mathcal{H}_{R}^{(j+1)} \neq\{0\}$, but $\mathcal{H}_{R}^{(j+1)} \subsetneq \mathcal{H}_{R}^{(j)}$, define $\mathcal{H}_{T}^{(j+1)}$ as the orthogonal complement of $\mathcal{H}_{R}^{(j+1)}$ in $\mathcal{H}_{R}^{(j)}$, that is, $\mathcal{H}_{R}^{(j+1)}=\mathcal{H}_{R}^{(j)} \ominus$ $\mathcal{H}_{R}^{(j+1)}$.

c. If $\mathcal{H}_{R}^{(j+1)}=\mathcal{H}_{R}^{(j)}$ (that is, $L_{P, k}^{(j)}=0 \forall k$ ), define

$$
\tilde{\mathcal{L}}_{P}^{(j)}:=-i H_{P}^{(j)}-\frac{1}{2} \sum_{k} L_{Q, k}^{(j) \dagger} L_{R, k}^{(j)} .
$$

- If $\tilde{\mathcal{L}}_{P}^{(j)} \neq 0$, re-define $\mathcal{H}_{R}^{(j+1)}:=\operatorname{ker}\left(\tilde{\mathcal{L}}_{\mathrm{P}}^{(\mathrm{j})}\right)$. If $\mathcal{H}_{R}^{(j+1)}=\{0\}$, define $\mathcal{H}_{T}^{(j+1)}:=\mathcal{H}_{R}^{(j)}$ and the iterative procedure is successfully completed. Otherwise define $\mathcal{H}_{T}^{(j+1)}:=$ $\mathcal{H}_{R}^{(j)} \ominus \mathcal{H}_{R}^{(j+1)}$.

- If $\tilde{\mathcal{L}}_{P}^{(j)}=0$, then $\mathcal{H}_{R}^{(j)}$ is invariant and $\mathcal{H}_{S}$ cannot be GAS. Exit the algorithm.

4. Define $\mathcal{H}_{S}^{(j+1)}:=\mathcal{H}_{S}^{(j)} \oplus \mathcal{H}_{T}^{(j+1)}$. To construct a basis for $\mathcal{H}_{S}^{(j+1)}$, append to the already defined basis for $\mathcal{H}_{S}^{(j)}$ an orthonormal basis for $\mathcal{H}_{T}^{(j+1)}$.

5. Increment the counter $j$ and go back to step 1.

The algorithm ends in a finite number of steps, since at every iteration it either stops or the dimension of $\mathcal{H}_{R}^{(j)}$ is reduced by at least one. 